\title{
Conformal geodesics on gravitational instantons
}

\author{
BY MACIEJ DUNAJSKI \\ Department of Applied Mathematics and Theoretical Physics, \\ University of Cambridge, \\ Wilberforce Road, Cambridge CB3 OWA. \\ e-mail: m.dunajski@damtp.cam.ac.uk \\ AND PAUL TOD \\ The Mathematical Institute, Oxford University, \\ Woodstock Road, Oxford OX2 6GG. \\ e-mail:tod@maths.ox.ac.uk
}

(Received 23 October 2019; revised 17 May 2021; accepted 17 May 2021)

\begin{abstract}
We study the integrability of the conformal geodesic flow (also known as the conformal circle flow) on the $S O(3)$-invariant gravitational instantons. On a hyper-Kähler four-manifold the conformal geodesic equations reduce to geodesic equations of a charged particle moving in a constant self-dual magnetic field. In the case of the anti-self-dual Taub NUT instanton we integrate these equations completely by separating the Hamilton-Jacobi equations, and finding a commuting set of first integrals. This gives the first example of an integrable conformal geodesic flow on a four-manifold which is not a symmetric space. In the case of the Eguchi-Hanson we find all conformal geodesics which lie on the threedimensional orbits of the isometry group. In the non-hyper-Kähler case of the Fubini-Study metric on $\mathbb{C P}^{2}$ we use the first integrals arising from the conformal Killing-Yano tensors to recover the known complete integrability of conformal geodesics.
\end{abstract}

2020 Mathematics Subject Classification: 53C18 (primary).

\section{Introduction}

The geodesic flow on a (pseudo) Riemannian manifold $(M, g)$ is integrable if the underlying metric admits a sufficient number of Killing vectors, or Killing tensors. The former correspond to first integrals linear in the velocity, and the latter give polynomial first integrals of higher order. For example the geodesic motion on a round two-sphere is integrable as any generator of the isometry group $S O(3)$ gives a Killing vector commuting with the Hamiltonian. On a tri-axial ellipsoid there are no Killing vectors, but the additional quadratic first integral required for integrability is given by a rank-two Killing tensor [18].

(C) The Author(s), 2021. Published by Cambridge University Press on behalf of Cambridge Philosophical Society. This is an Open Access article, distributed under the terms of the Creative Commons Attribution licence (http:// creativecommons.org/licenses/by/4.0/), which permits unrestricted re-use, distribution, and reproduction in any medium, provided the original work is properly cited. 
Let $u$ be a unit tangent vector to a curve $\Gamma \subset M$, and let $a \equiv \nabla_{u} u$ be the acceleration of $\Gamma$. The geodesic condition $a=0$ is not invariant under conformal rescalings of the metric $g \rightarrow \Omega^{2} g$, but there is a different preferred set of curves on manifolds endowed with only conformal structures $(M,[g])$, where $[g]=\left\{\Omega^{2} g, \Omega: M \rightarrow \mathbb{R}^{+}\right\}$. These conformal geodesics are also known as conformal circles, and arise as solutions to a system of third order ODEs on $M$. A conformal geodesic $\Gamma$ is uniquely specified by a point, a tangent direction, and a perpendicular acceleration. If $g$ is a representative metric in the conformal class, and $\nabla$ is the Levi-Civita connection of $g$, then the conformal geodesic equations are

$$
\nabla_{u} a=-\left(|a|^{2}+L(u, u)\right) u+L^{\sharp}(u),
$$

where $L \in \Gamma(T M \otimes T M)$ is the Schouten tensor given in terms of the Ricci tensor $R$ and the Ricci scalar $S$ by

$$
L=\frac{1}{n-2}\left(R-\frac{1}{2(n-1)} S g\right),
$$

and $L^{\sharp}: T M \rightarrow T M$ is the endomorphism defined by $g\left(L^{\sharp}(X), Y\right)=L(X, Y)$ for all vector fields $X, Y$. It can be demonstrated $[3,4,24,25]$ that the conformal geodesics only depend on the conformal class of $[g]$, and not on the choice of the representative metric. In (1.2) it is assumed that $n>2$. The case where $n=2$ will be discussed in Section 2.2.2.

Neither the Killing vectors nor the conformal Killing vectors of $g$ give rise to first integrals of $(1 \cdot 1)$, and it is natural to ask whether there are examples of integrable conformal geodesic motions, and what geometric structures on $(M,[g])$ give rise to this integrablity. In $[\mathbf{1 6}, \mathbf{2 5}]$ it was shown that the conformal Killing-Yano two-forms (CKY) give rise to first integrals of (1·1): $Y \in \Lambda^{2}\left(T^{*} M\right)$ is a CKY if $(\nabla Y)_{0} \in \Gamma\left(\Lambda^{3}\left(T^{*} M\right)\right)$, where $T_{0}$ denotes the trace-free part of $T$. The corresponding first integral is then

$$
Q=Y(u, a)-\frac{1}{n-1} \operatorname{div}(Y)(u),
$$

where $n=\operatorname{dim}(M)$, and $\operatorname{div}=* d *$ is the divergence. This was sufficient [25] to integrate (1-1) on a non-conformally flat Nil 3-manifold, and a squashed 3-sphere but already the conformal class of the Schwarzchild metric proved too difficult to handle, as there do not exist sufficiently many CKYs.

In this paper we shall study the integrability of $(1 \cdot 1)$ on four-dimensional conformal structures corresponding to some gravitational instantons: solutions to Einstein equations on Riemannian four-manifolds which are compact or complete metrics which asymptotically approach a locally flat space - the decay rate, as well as the topology at infinity varries between different examples of instantons. See [7, 13] for details.

We shall explore the existence of three CKYs corresponding to the underlying hyperKähler structure, and reduce (1.1) to a system of 2nd order ODEs corresponding to a forced geodesic equation in a constant magnetic field. Further progress can be made for the antiself-dual (ASD) Taub-NUT and the Eguchi-Hanson instantons. In the ASD Taub-NUT case we shall establish complete integrability by separating the Hamilton-Jacobi equation.

THEOREM 1.1. The conformal geodesic equation on the anti-self-dual Taub-NUT manifold is completely integrable in the Arnold-Liouville sense: it reduces to a geodesic equation in a self-dual magnetic field which admits four first integrals in involution. 
The associated Hamilton-Jacobi equation is separable, and the conformal geodesic equations reduce to quadratures.

The Eguchi-Hanson metric admits an isometric and tri-holomorphic action of $S O(3)$ which preserves the magnetic field, and therefore gives rise to three charged linear first integrals of the Lorentz force equation. While full integrablity cannot be established in this case, there is enough structue to reduce the conformal geodesic motion to quadratures under an additional assumption that the conformal geodesics lie on the 3-dimensional orbits of the $S O(3)$ subgroup of the isometry group.

The paper is organised as follows. In the next section we shall introduce the conformal geodesic equations (1.1), and focus on the special case where the underlying conformal structure admits an Einstein metric. We shall discuss the first-integrals of (1.1), and establish a link with the Lorentz force equations for hyper-Kähler four manifolds (Proposition 3.1). In Section 4 and 5 we shall study (1.1) on the ASD Taub-NUT metric, and the Eguchi-Hanson metric respectively. The ASD Taub-NUT case is completely integrable (Theorem 1.1 will follow from Proposition 4.1 and Proposition 4.2), and the Eguchi-Hanson case has a couple of integrable sub-cases (Proposition 5.1).

In Section 6 we shall establish the integrability of the conformal geodesic flow for the Fubini-Study metric on $\mathbb{C P}^{2}$. We shall establish it by making use of nine CKYs on this space, and recover the results of [2], where all conformal geodesics of $\mathbb{C P}^{n}$ have been characterised by their horizontal lifts to helices on the total space of the fibration $S^{2 n+1} \rightarrow \mathbb{C P}^{n}$. In Appendix A we shall rule out the existence of non-flat Riemannian Gibbons-Hawking metrics with three commuting vector fields, and in Appendix B we shall provide necessary and sufficient conditions for a Killing trajectory to be a conformal geodesic.

\section{Conformal geodesics on Einstein manifolds}

Let $(M, g)$ be a Riemannian manifold. When using the index-free notation we shall denote the $g$-inner product of two vector fields $X$ and $Y$ by $g(X, Y)$. We also set $|X|^{2} \equiv g(X, X)$, and use notation $X\lrcorner \psi$ for the ( $p-1)$-form arising as a contraction of the $p$-form $\psi$ with the vector field $X$. If $\Gamma$ is a curve, and $u$ is a tangent vector to $\Gamma$, then $\nabla_{u}$ denotes the directional derivative along $\Gamma$, where $\nabla$ is the Levi-Civita connection of $g$.

In explicit computations involving conformal geodesics, and conformal KillingYano tensors it is convenient to adopt the abstract index notation [23]. Thus $u^{a}, a=$ $1,2, \ldots, \operatorname{dim}(M)$ denotes a vector, $u^{a} \nabla_{a} v^{b}$ denotes the directional covariant derivative $\nabla_{u} v$ of another vector $v$. On a Riemannian manifold $(M, g)$ an isomorphism between $T M$ and $T^{*} M$ is realised by $u_{a}=g_{a b} u^{b}$, where now $u_{a}$ denotes a one-form, and the Einstein summation convention is used. Despite the presence of these indices, no choice of basis has been made.

If the metric $g$ is Einstein, i.e. $R=(S / n) g$ then the conformal geodesic equation (1-1) for a curve $\Gamma$ parametrised by an arc-lengh $s$, and with a unit tangent vector $u$ reduces to

$$
\nabla_{u} a=-|a|^{2} u, \quad \text { where } \quad|a|^{2} \equiv g(a, a) \geq 0 \text { is a constant, } \quad \text { and } \quad g(a, u)=0 .
$$

This system of 3rd order equations (2.4) has long been studied in Riemannian geometry $[2,22]$, where the corresponding solution curves have been called circles. The terminology 
is motivated by the observation that a development ${ }^{1}$ of a circle $\Gamma$ starting at a point $x \in M$ is an ordinary circle in $T_{x} M$. It is also the case that circles on the round sphere $S^{n}$ are intersections of $S^{n} \subset \mathbb{R}^{n+1}$ with planes (not necessarily through the origin) in $\mathbb{R}^{n+1}$. Thus they are indeed circles.

\subsection{First integrals}

If $Y \in \Lambda^{2}(M)$ satisfies the conformal Killing-Yano (CKY) equation

$$
\nabla_{a} Y_{b c}=\nabla_{[a} Y_{b c]}-2 g_{a[b} K_{c]}
$$

for some one-form $K \in \Lambda^{1}(M)$, then (if $\operatorname{dim}(M)=4$ ) equation (1.1) implies that

$$
Q=u^{a} a^{b} Y_{a b}-u^{a} K_{a}
$$

is constant along the conformal geodesics. In the special case where $Y_{a b}$ is a Kähler form, the one form $K$ is zero and the linear term in (2.6) is not present. In this case the first integral (2.6) has been called the complex torsion (though it is real) in [2]. In four dimensions the condition $u^{c} \nabla_{c} Q=0$ with $Q$ given by (2.6) was established in [25]. In [16] it was put in the general context of parabolic geometries.

\subsection{Examples}

\subsubsection{Circles on $\mathbb{R}^{n}$.}

These are just ordinary circles. Equation (2.4) becomes $\dddot{\mathbf{x}}=-|a|^{2} \dot{\mathbf{x}}$, and can be readily solved

$$
\mathbf{x}(s)=\mathbf{x}(0)+|a|^{-1} \mathbf{v}(0) \sin (|a| s)+|a|^{-2} \mathbf{a}(0)(1-\cos (|a| s)),
$$

where $\mathbf{x}(0)$ is arbitrary, $\mathbf{v}(0)$ is a unit vector in $\mathbb{R}^{n}$, and the vector $\mathbf{a}(0)$ is orthogonal to $\mathbf{v}(0)$, and has squared norm $|a|^{2}$. We recognise these conformal geodesics as circles centered at $\mathbf{x}(0)+|a|^{-2} \mathbf{a}(0)$, and with Euclidean radius $|a|^{-1}$.

Conformal rescalings of the metric preserve conformal geodesics, so circles on $S^{n}$ are also circles (intersections of $S^{n} \subset \mathbb{R}^{n+1}$ with planes in $\mathbb{R}^{n+1}$ which do not necessarily pass through the origin). In particular all conformal geodesics on $S^{n}$ are closed.

\subsubsection{Magnetic motion on the upper half-plane.}

Conformal geodesics on the hyperbolic space can also be obtained from those on $\mathbb{R}^{n}$. This time however they are not necessarily closed, and we shall consider this case separately to illustrate the significance of the first integrals in the qualitative behaviour of conformal geodesics.

\footnotetext{
${ }^{1}$ In references [2, 22] the equation (2.4) is written as $\nabla_{s} X_{S}=|a| Y_{S}, \quad \nabla_{S} Y_{S}=-|a| X_{S}$ where $X_{S}$ and $Y_{S}$ are unit vector fields, $X_{s}$ is the tangent vector to the curve $\Gamma$ parametrised by the arc-length $s, \nabla_{s}$ is the covariant derivative of $g$ along $\Gamma$ and the positive constant $|a|^{-1}$ is the radius of the circle $\Gamma$.

If $\Gamma_{0}^{s}$ is the parallel displacement of tangent vectors along $\Gamma$ from $\Gamma(s)$ to $\Gamma(0)$, and $X_{s}{ }^{*}=\Gamma_{0}^{s}\left(X_{s}\right)$, then the development $\Gamma^{*}$ is the unique curve in $T_{x} M$ starting at the origin such that its tangent vector is parallel to $X_{s}$ in the Euclidean sense.
} 


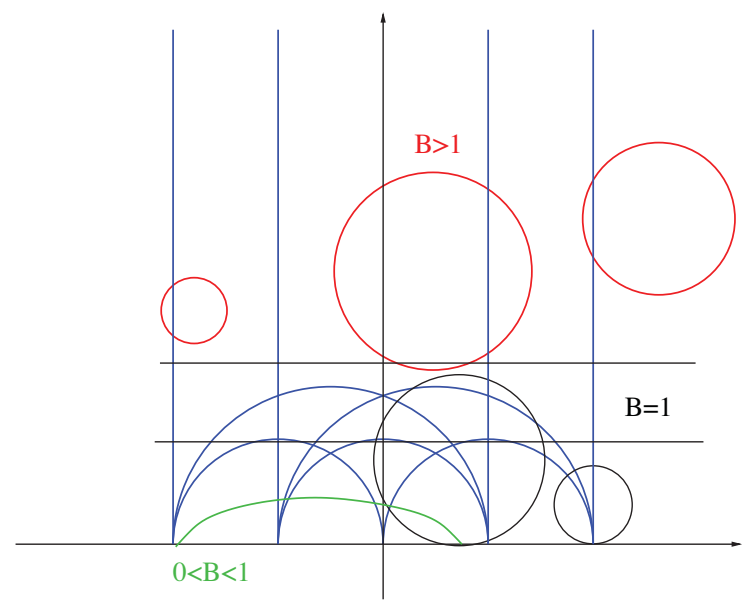

Fig. 1. Geodesics (in blue) and circles on $\mathbb{H}$.

Let $\mathbb{H}$ be the upper half-plane with coordinates $(x, y)$, and a constant curvature metric

$$
g=\frac{d x^{2}+d y^{2}}{y^{2}}, \quad y>0
$$

(so the Ricci scalar equal to -2 ) and a magnetic field $F=B \operatorname{vol}_{\mathbb{H}}$ given by a constant $B>0$ multiple of the parallel volume form on $\mathbb{H}$. We chose the potential $\Phi=B y^{-1} d x$, so that $F=d \Phi$. The magnetic Lagrangian ${ }^{2}$

$$
L=\frac{1}{2} \frac{\dot{x}^{2}+\dot{y}^{2}}{y^{2}}-B \frac{\dot{x}}{y}
$$

admits a conserved energy integral which we set to 1 . Taking $u$ to be a unit tangent vector to an integral curve of the corresponding Euler-Lagrange equations we find

$$
a \equiv \nabla_{u} u=B J(u),
$$

where $J$ is the complex structure on $\mathbb{H}$ defined by $g(v, J(w))=\operatorname{vol}_{\mathbb{H}}(v, w)$. Therefore the circle equations (2.4) hold with $|a|^{2}=B^{2}$, as

$$
\nabla_{u} a=B \nabla_{u} J(u)=B J\left(\nabla_{u} u\right)=B^{2} J^{2}(u)=-B^{2} u .
$$

The two first integrals $|a|^{2}$, and $Q$ given by (2.6) (where now $Y=F$ ) are therefore equal as

$$
Q \equiv F(u, a)=B \operatorname{vol}_{\mathbb{H}}(u, B J(u))=B^{2} g(u, u)=B^{2} .
$$

The resulting trajectories are circles of radius $|B|^{-1}$. The behaviour of the circles depends on the value of $B$ (Figure 1)

The circles are open (in the language of [6] the magnetic field is not strong enough to capture the particle) and unbounded if $0<B<1$, and closed if $B>1$. The special case $B=1$ corresponds to horocircles tangent to the $y=0$ boundary of $\mathbb{H}$, or lines $y=$ const parallel to the $x$-axis.

${ }^{2}$ The variational formulation of the conformal geodesic equations with $n>2$ has been developed in [8]. 


\section{Circles on hyper-Kähler four-manifolds and the Lorentz force}

Let $(M, g)$ be an oriented Riemannian-four manifold with a hyper-Kähler structure given by parallel two-forms $\Omega^{1}, \Omega^{2}, \Omega^{3}$. In particular $(M, g)$ is Ricci-flat, so the conformal geodesic equations reduce to $(2 \cdot 4)$.

PROPOSITION 3.1. The conformal geodesic equations on a hyper-Kähler four-manifold $(M, g)$ reduce to the Lorentz force equations

$$
\nabla_{u} u=|a| J(u),
$$

where $J$ is the complex structure corresponding to the Kähler form given by a constant self-dual Maxwell field $F=\sum_{j} c^{j} \Omega^{j}$ with constants $\left(c^{1}, c^{2}, c^{3}\right)$ such that $|\mathbf{c}|=1$.

Proof. Each of the Kähler forms gives rise to a first integral (2.6), so there are three of those. These three integrals allow us to integrate the circle equations once, and reduce them, on a level set

$$
c^{i}=\Omega^{i}(u, a), \quad i=1,2,3,
$$

to geodesic equations of a charged particle moving in a magnetic field.

If the orientation of $(M, g)$ is chosen so that the Riemann tensor of $g$ is anti-self-dual, then this magnetic field is self-dual. To see how the Lorentz force arises assume that $M$ is paralelisable chose an orthonormal frame such that

$$
g=\left(e^{1}\right)^{2}+\left(e^{2}\right)^{2}+\left(e^{3}\right)^{2}+\left(e^{4}\right)^{2},
$$

and

$$
\Omega^{1}=e^{1} \wedge e^{4}+e^{2} \wedge e^{3}, \quad \Omega^{2}=e^{2} \wedge e^{4}+e^{3} \wedge e^{1}, \quad \Omega^{3}=e^{3} \wedge e^{4}+e^{1} \wedge e^{2} .
$$

Let $u^{a}$ and $a^{a}$ be the components of $u, a$ in the frame of vector-fields dual to $e^{a}$. The relations (3.8) can then be solved for $a$. Using the ordinary vector notation for the components $\mathbf{u}=$ $\left(u^{1}, u^{2}, u^{3}\right), \mathbf{a}=\left(a^{1}, a^{2}, a^{3}\right)$ we can rewrite (3.8) as $\mathbf{c}=\mathbf{u} a^{4}-\mathbf{a} u^{4}+\mathbf{u} \wedge \mathbf{a}$, and find

$$
\mathbf{a}=\mathbf{c} \wedge \mathbf{u}-u^{4} \mathbf{c}, \quad a^{4}=\mathbf{u} \cdot \mathbf{c} .
$$

Note that

$$
g(a, u)=\mathbf{a} \cdot \mathbf{u}+a^{4} u^{4}=0, \quad g(a, a)=|\mathbf{c}|^{2}
$$

so the first integrals $g(a, a)$ and $\mathbf{c}$ are not independent. The circle equations now reduce to $a=\nabla_{u} u$, or (3.7) which is the Lorentz force equation for a unit mass particle with charge $e=|a|$ in a constant self-dual Maxwell field

$$
F=\frac{1}{|a|}\left(c^{1} \Omega^{1}+c^{2} \Omega^{2}+c^{3} \Omega^{3}\right) .
$$

In view of (3.10) we can redefine $\mathbf{c}$ to be a unit-vector, and recover the statement of Proposition $3 \cdot 1$.

For an uncharged particle $|a|=0$, and (3.7) reduces to the ordinary geodesic equation. To make further progress with $|a| \neq 0$ we need to seek first integrals of (3.7). Any Killing vector on $(M, g)$ which is also tri-holomorphic, i .e. $\mathcal{L}_{K} \Omega^{i}=0$, will give rise to a first integral: If 
$F=d \Phi$, the potential one-form $\Phi$ can be chosen such that $\mathcal{L}_{K} \Phi=0$ (we then say that $K$ Lie drags $\Phi)$. Indeed, computing

$$
\left.\left.0=\mathcal{L}_{K} F=d(K\lrcorner F\right) \quad \text { implies that, locally } \quad K\right\lrcorner F=d f
$$

for some function $f: M \rightarrow \mathbb{R}$. Now

$$
\left.\mathcal{L}_{K} \Phi=d(f+K\lrcorner \Phi\right)
$$

Choosing $f_{1}: M \rightarrow \mathbb{R}$ such that $\left.K\left(f_{1}\right)=-f-K\right\lrcorner \Phi$, and performing a gauge transformation $\Phi \rightarrow \Phi+d f_{1}$ we establish that in this gauge $\mathcal{L}_{K} \Phi=0$. This implies that

$$
\mathcal{K}:=K^{a}\left(u_{a}+e \Phi_{a}\right)=K^{a} p_{a}
$$

is conserved along conformal geodesics on a level set of the $c^{i}$. The momentum $p_{a}$ is defined by

$$
u^{a}=g^{a b}\left(p_{a}-e \Phi_{a}\right)
$$

which is the usual convention for charged particles.

For (3.7) to be integrable we in particular need the geodesic equation to be integrable, and we need four constants of the motion in involution. One is the Hamiltonian (the unit mass) and the other three need to come from Killing vectors and Killing tensors. If at least two of them arise from Killing vectors then, to be involutive, these must commute and to give integrals for (3.7) they must Lie-drag $\Omega^{i}$. Now necessarily there is a constant linear combination of them which has purely anti-self-dual covariant derivative (this is $[\mathbf{1 5}$, Theorem $2 \cdot 3$ ], credited to Hitchin by these authors) and this is the characterising property of the Gibbons-Hawking metrics [13]. Consequently we henceforth restrict to these metrics, but there may be other integrable cases with two or more Killing tensors (see $[12,17]$ for other applications for Yano tensors to geodesic integrability).

\subsection{The Gibbons Hawking metrics}

All hyper-Kähler four-manifolds which admit a tri-holomorphic Killing vector can be put in the Gibbons-Hawking form [13]

$$
g=V \mathbf{d x} \cdot \mathbf{d x}+V^{-1}(d \tau+\omega)^{2},
$$

where $(V, \omega)$ is a function, and a one-form on $\mathbb{R}^{3}$. Choosing a frame

$$
e^{4}=V^{-1 / 2}(d \tau+\omega), \quad e^{i}=V^{1 / 2} d x^{i}, \quad E_{4}=V^{1 / 2} \partial_{\tau}, \quad E_{i}=V^{-1 / 2}\left(\partial_{i}-A_{i} \partial_{\tau}\right)
$$

one verifies that the two-forms $\Omega^{i}$ are closed iff

$$
d \omega=* d V
$$

which in particular implies that $V$ is harmonic.

Consider the Killing vector $K=\partial_{\tau}$ of metric (3.12), and set $K^{\mathrm{b}}=V^{-1}(d \tau+\omega)$ and then its derivative is anti-self-dual (the characterising property of the Gibbons-Hawking metrics). It follows that $K$ is tri-holomorphic, i. e.

$$
\mathcal{L}_{K} \Omega^{i}=0
$$


where $\mathcal{L}_{X}$ is the Lie-derivative along the vector field $X$. It therefore follows that $K$ gives rise to three moment maps: for each $i$ there is a function $x^{i}$ satisfying

$$
K\lrcorner \Omega^{i}=d x^{i}
$$

and these $x^{i}$ are (up to scale) the flat coordinates $(x, y, z)$ in (3.12).

The Killing vector $K$ will give a constant of the motion (3.11) for (3.7). This is conserved for any $V$, and we will return to it when we consider examples below. It is however not sufficient for integrability.

To integrate (3.7) we need either two more Killing vectors, commuting with each other and with $K=\partial_{\tau}$, or another Killing vector commuting with $K$ and a Killing tensor with suitably involutive. The first case is not interesting (see the Appendix A) but for the second we can take motivation from the familiar fact that both the self-dual Taub-NUT metric and the Eguchi-Hanson metric admit Killing tensors [15] to concentrate on these two metrics in Sections 4 and 5.

\section{Circles on anti-self-dual Taub-NUT}

The ASD Taub-NUT metric [13] is a special case of (3.12) with $V=1+m / r$ where $m$ is a non-negative constant, and (using spherical polar coordinates on $\mathbb{R}^{3}$ ) $\omega=m \cos \theta d \phi$ and

$$
g=\left(1+\frac{m}{r}\right)\left(d r^{2}+r^{2}\left(d \theta^{2}+\sin ^{2} \theta d \phi^{2}\right)\right)+\left(1+\frac{m}{r}\right)^{-1}(d \psi+m \cos \theta d \phi)^{2}
$$

writing $\psi$ for $\tau$ to emphasise the Bianchi IX $S U$ (2) symmetry. The singularity at $r=0$ is removable. The infinity $r \rightarrow \infty$ has the topology of a one-monopole $S^{1}$ bundle over $S^{2}$. This behaviour is referred to as asymptotic local flatness (ALF). Thus ASD Taub-NUT is regular everywhere, and is an example of a gravitational instanton.

The metric has $S U(2) \times U(1)$ as its group of isometries where the $U(1)$ action generated by $\partial / \partial \psi$ is tri-holomorphic, i. e.

$$
\mathcal{L}_{\partial / \partial \psi} \Omega^{i}=0, \quad i=1,2,3
$$

and so the self-dual derivative of the corresponding one-form $g(\partial / \partial \psi, \cdot)=V^{-1}(d \psi+$ $\cos \theta d \phi$ ) vanishes. The $S U(2)$ action is not tri-holomorphic, and the exterior derivatives of the corresponding Killing vectors do not have a definite duality (but their SD derivatives are parallel). However for any unit vector $\mathbf{c} \in \mathbb{R}^{3}$ there exists a linear combination $L$ of the isometric generators of $S U(2)$, such that $L$ Lie-drags $\sum_{i} c^{i} \Omega^{i}$.

\subsection{Conformal Killing-Yano tensors and Arnold-Liouville integrability}

The ASD Taub-NUT metric admits a Killing-Yano 2-form $Z$, i. e. a two-form such that

$$
\nabla_{a} Z_{b c}=\nabla_{[a} Z_{b c]} .
$$

The self-dual, and anti-self-dual parts of $Z$ are conformal Killing-Yano two-forms which satisfy $(2 \cdot 5)$ :

$$
\begin{aligned}
Z & =(d \psi+m \cos \theta d \phi) \wedge d r+(2 r+m)(r+m) r \sigma_{1} \wedge \sigma_{2} \\
& =Y-W, \quad \text { where } \quad * Y=Y, \quad * W=-W
\end{aligned}
$$


and

$$
Y=\frac{r^{3}}{m} d(V(d \psi+m \cos \theta d \phi)), \quad W=-\frac{(r+m)^{3}}{m} d\left(V^{-1}(d \psi+m \cos \theta d \phi)\right) .
$$

The left invariant one-forms $\sigma_{i}, i=1,2,3$ on the group manifold $S U(2)$ are such that

$$
d \sigma_{1}+\sigma_{2} \wedge \sigma_{3}=0, \quad d \sigma_{2}+\sigma_{3} \wedge \sigma_{1}=0, \quad d \sigma_{3}+\sigma_{1} \wedge \sigma_{2}=0 .
$$

These one-forms can be represented in terms of Euler angles by

$$
\sigma_{1}+i \sigma_{2}=e^{-i \psi}(d \theta+i \sin \theta d \phi), \quad \sigma_{3}=d \psi+\cos \theta d \phi,
$$

where to cover $S U(2)=S^{3}$ we require the ranges $0 \leq \theta \leq \pi, \quad 0 \leq \phi \leq 2 \pi, \quad 0 \leq \psi \leq 4 \pi$. Up to a multiple of 4 the two-form $Z$ coincides with the Killing-Yano two-from found by Gibbons and Ruback [15] (see formula (2.12) in this reference with $n=1$, and make a coordinate transformation $t=m+2 r$. See also [26]).

Both $Y$ and $W$ satisfy $(2 \cdot 5)$ with the same Killing vector $K=g(\partial / \partial \psi, \cdot)$. The CKY twoform $Y$ was discovered in [10], where it was used to show that the ASD Taub-NUT metric is conformal to a scalar-flat Kähler metric with a non-constant conformal factor. Computing the first integral (2.6) corresponding to $Y$ gives an expression which is functionally dependent with the first integrals arising from the Killing vectors $K=\partial / \partial \psi$ and $L=\partial / \partial \phi$, so it does not give anything useful. We instead turn to $W$, where the resulting first integal (2.6) is non-trivial, but it does not in general commute with the first integrals (3.11) arising from $\partial_{\psi}$ and $\partial_{\phi}$. To get around this problem we proceed as follows:

Given (3.7) with some $F$, we may first rotate the $(x, y, z)$ coordinates until $F=-\Omega^{3}$ : if this case is integrable then the general case is integrable. Introducing spherical polar coordinates $(r, \theta, \phi)$ in place of $(x, y, z)$ yields

$$
\begin{aligned}
F & =-\Omega^{3}=d \Phi \quad \text { with } \\
\Phi & =-z(d \psi+\omega)+\left(\frac{m}{r}+\frac{1}{2}\right)(y d x-x d y) \\
& =-r \cos \theta d \psi-\left(m r+\frac{1}{2} r^{2} \sin ^{2} \theta\right) d \phi
\end{aligned}
$$

or in polars and with the index raised

$$
\Phi^{\#}=-z V \partial_{\psi}+\frac{1}{V} \partial_{\phi}
$$

In this form it is clear that $\Phi^{\#}$ commutes with the Killing vectors $K:=\partial_{\psi}$ and $L:=\partial_{\phi}$, which in turn commute with each other, leading to two constants of the motion for (3.7).

This leads to four independent first integrals $\mathcal{I}_{a}=(\mathcal{K}, \mathcal{L}, \mathcal{W}, \mathcal{H})$ two of which are linear in the velocity, and two of which are quadratic:

$$
\begin{aligned}
& \mathcal{K}=K^{a}\left(u_{a}+e \Phi_{a}\right), \quad \mathcal{L}=L^{a}\left(u_{a}+e \Phi_{a}\right), \\
& \mathcal{H}=\frac{1}{2} g_{a b} u^{a} u^{b}, \quad \mathcal{W}=e W_{a c} F_{b}^{c} u^{a} u^{b}-2 \mathcal{H} K^{a} u_{a} .
\end{aligned}
$$

To complete the calculation we shall use Hamiltonian formalism. In the presence of a magnetic field there are two ways to proceed starting from the magnetic Lagrangian $\mathcal{G}=$ (1/2) $g_{a b} u^{a} u^{b}+e \Phi_{b} u^{b}$. One way is to define the conjugate momentum 


$$
p_{a}=\frac{\partial \mathcal{G}}{\partial u^{a}}=u_{a}+e \Phi_{a}
$$

and perform a Legendre transform to find four first integrals

$$
\begin{aligned}
\mathcal{K} & =p_{\psi}, \quad \mathcal{L}=p_{\phi}, \\
\mathcal{H} & =\frac{1}{2} g^{a b}\left(p_{a}-e \Phi_{a}\right)\left(p_{b}-e \Phi_{b}\right), \\
\mathcal{W} & =e W^{a}{ }_{c} F^{c b}\left(p_{a}-e \Phi_{a}\right)\left(p_{b}-e \Phi_{b}\right)-2 \mathcal{H}\left(\mathcal{K}-e K^{a} \Phi_{a}\right) .
\end{aligned}
$$

These are in involution with respect to the standard symplectic structure on $T^{*} M$ given by $d p_{a} \wedge d q^{a}$, where $q^{a}=(r, \phi, \theta, \psi)$ and $p_{a}=\left(p_{r}, p_{\phi}, p_{\theta}, p_{\psi}\right)$. Alternatively define a momentum by

$$
P_{a}=p_{a}-e \Phi_{a},
$$

and consider the charged symplectic form

$$
\Omega=d P_{a} \wedge d q^{a}+e F
$$

on the 8-dimensional phase-space with coordinates $q^{a}=(r, \phi, \theta, \psi)$ and $P_{a}=$ $\left(P_{r}, P_{\phi}, P_{\theta}, P_{\psi}\right)$. The set of first integrals is then $\mathcal{I}_{a}=(\mathcal{K}, \mathcal{L}, \mathcal{H}, \mathcal{W})$, where

$$
\begin{aligned}
\mathcal{K}= & P_{\psi}-e r \cos \theta, \\
\mathcal{L}= & P_{\phi}-e\left(m r+\frac{1}{2} r^{2} \sin ^{2} \theta\right), \\
\mathcal{H}= & \frac{1}{2}\left(\frac{r}{r+m} P_{r}^{2}+\frac{1}{r(r+m)} P_{\theta}{ }^{2}+\frac{r+m}{r} P_{\psi}{ }^{2}+\frac{1}{r(r+m) \sin ^{2} \theta}\left(P_{\phi}-m \cos \theta P_{\psi}\right)^{2}\right), \\
\mathcal{W}= & \frac{e \cos \theta}{r}\left(r P_{r}-\tan \theta P_{\theta}\right)^{2}-\frac{e}{r \cos \theta} P_{\theta}{ }^{2} \\
& -\frac{e \cos \theta}{r \sin ^{2} \theta}\left(P_{\phi}{ }^{2}+\left(m^{2}-r^{2} \sin ^{2} \theta\right) P_{\psi}{ }^{2}-2 \frac{m+r \sin ^{2} \theta}{\cos \theta} P_{\psi} P_{\phi}\right)-2 \mathcal{H} P_{\psi} .
\end{aligned}
$$

These integrals are in involution

$$
\left\{\mathcal{I}_{a}, \mathcal{I}_{b}\right\}=0, \quad a, b=1, \ldots, 4
$$

with respect to Poisson brackets of the charged symplectic structure (4.18). We have established:

PROPOSITION 4.1. The conformal geodesic equation on the anti-self-dual Taub-NUT manifold is completely integrable in the sense of Arnold-Liouville.

Rather than finding the action-angle variables explicitly, we follow [15] and seek to separate the Hamilton-Jacobi equation, but for (3.7) rather than for the geodesic equation. 
4.2. Separating the Hamilton-Jacobi equation for anti-self-dual Taub-NUT

Following Gibbons-Ruback [15], we introduce parabolic coordinates

$$
\eta=r(1+\cos \theta), \quad \xi=r(1-\cos \theta), \phi=\phi,
$$

so that in the cylindrical polars obtained from $(x, y, z)$ in $(3 \cdot 12)$

$$
z=\frac{1}{2}(\eta-\xi), \quad \rho=\sqrt{\eta \xi}, \phi=\phi
$$

Now

$$
z+i \rho=\frac{1}{2}(\sqrt{\eta}+i \sqrt{\xi})^{2}
$$

and

$$
\begin{gathered}
d z^{2}+d \rho^{2}=\frac{1}{4}(\eta+\xi)\left(\frac{d \eta^{2}}{\eta}+\frac{d \xi^{2}}{\xi}\right), \\
V=1+\frac{m}{r}=\frac{(\eta+\xi+2 m)}{(\eta+\xi)}, \quad \omega=m \cos \theta d \phi=m \frac{(\eta-\xi)}{(\eta+\xi)} d \phi,
\end{gathered}
$$

so that the anti-self-dual Taub-NUT metric is

$$
g=V\left(\frac{1}{4}(\eta+\xi)\left(\frac{d \eta^{2}}{\eta}+\frac{d \xi^{2}}{\xi}\right)+\eta \xi d \phi^{2}\right)+\frac{1}{V}\left(d \psi+m \frac{(\eta-\xi)}{(\eta+\xi)} d \phi\right)^{2} .
$$

For the geodesic equation first, we seek Hamilton's Principal Function $S$ in the separated form

$$
S=E \psi+J \phi+F(\xi)+G(\eta)
$$

with constant $E, J$ then the Hamilton-Jacobi equation is

$$
\mu^{2}=g^{a b} \nabla_{a} S \nabla_{b} S
$$

where $\mu$ is the conserved mass which we will always take to be one.

Algebra leads to

$$
\begin{aligned}
4\left(\eta G_{\eta}^{2}+\xi F_{\xi}^{2}\right)= & \mu^{2}(\eta+\xi+2 m)-J^{2}\left(\frac{1}{\eta}+\frac{1}{\xi}\right)+2 J E m\left(-\frac{1}{\eta}+\frac{1}{\xi}\right) \\
& -E^{2}\left(\eta+\frac{m^{2}}{\eta}+\xi+\frac{m^{2}}{\xi}+4 m\right),
\end{aligned}
$$

and, confirming [15], this separates with a new constant $Q$ :

$$
\begin{aligned}
& 4 \eta G_{\eta}^{2}=Q+\mu^{2}(\eta+m)-\frac{J^{2}}{\eta}-\frac{2 J E m}{\eta}-E^{2}\left(\eta+\frac{m^{2}}{\eta}+2 m\right), \\
& 4 \xi F_{\xi}^{2}=-Q+\mu^{2}(\xi+m)-\frac{J^{2}}{\xi}+\frac{2 J E m}{\xi}-E^{2}\left(\xi+\frac{m^{2}}{\xi}+2 m\right) .
\end{aligned}
$$


The constant $Q$ is quadratic in momenta and must be associated with a quadratic Killing tensor. To obtain the geodesics from $S$, we solve

$$
\dot{q}^{a}=g^{a b} p_{b}=g^{a b} \nabla_{b} S,
$$

so that

$$
\dot{\eta}=\frac{4 \eta}{\eta+\xi+2 m} G_{\eta}, \quad \dot{\xi}=\frac{4 \xi}{\eta+\xi+2 m} F_{\xi}, \quad p_{\phi}=J, \quad p_{\psi}=E .
$$

We shall now modify this separation procedure to include the Maxwell field present for conformal geodesic motion.

Proposition 4.2. The Hamilton-Jacobi equation for the conformal geodesics on antiself-dual Taub-NUT manifold is separable.

Proof. To include a Maxwell field, we follow the Hamilton-Jacobi formalism in the presence of electro-magnetic field (see e.g. [19]). The method is to suppose the that potential is $\Phi_{a}$, so that

$$
\dot{q}^{a}=g^{a b}\left(p_{b}-e \Phi_{b}\right) \quad \text { and } \quad H=\frac{1}{2} g^{a b}\left(p_{a}-e \Phi_{a}\right)\left(p_{b}-e \Phi_{b}\right) .
$$

Now set $p_{a}=\nabla_{a} S$ and take the Hamilton-Jacobi equation to be

$$
\mu^{2}=g^{a b}\left(\nabla_{a} S-e \Phi_{a}\right)\left(\nabla_{b} S-e \Phi_{b}\right) .
$$

For us

$$
\begin{aligned}
\Phi & =-z(d \psi+\omega)-r^{2} \sin ^{2} \theta\left(\frac{1}{2}+\frac{m}{r}\right) d \phi=-\frac{1}{2}(\eta-\xi) d \psi-\frac{1}{2}(\eta \xi+m(\eta+\xi)) d \phi, \\
& =X d \phi+Y d \psi, \text { say. }
\end{aligned}
$$

The changes to the Hamilton-Jacobi equation are

$$
J \longrightarrow J-e X, E \longrightarrow E-e Y,
$$

leading to

$$
\begin{aligned}
4\left(\eta G_{\eta}^{2}+\xi F_{\xi}^{2}\right)= & \mu^{2}(\eta+\xi+2 m)-J^{2}\left(\frac{1}{\eta}+\frac{1}{\xi}\right)+2 J E m\left(-\frac{1}{\eta}+\frac{1}{\xi}\right) \\
& -E^{2}\left(\eta+\frac{m^{2}}{\eta}+\xi+\frac{m^{2}}{\xi}+4 m\right)-e J(\eta+\xi+4 m) \\
& -e E\left(\eta^{2}-\xi^{2}+3 m(\eta-\xi)\right) \\
& -\frac{e^{2}}{4}\left(\eta(\eta+2 m)^{2}+\xi(\xi+2 m)^{2}\right),
\end{aligned}
$$

which still separates with a quadratic constant and implied Killing tensor. As before, for the particle paths solve

$$
\dot{q}^{a}=g^{a b} p_{b}=g^{a b} \nabla_{b} S .
$$

Thus in anti-self-dual Taub-NUT the conformal geodesic equation is completely integrable: it admits four first integrals in involution established in Proposition 4.1, and the 
associated Hamilton-Jacobi equation is separable which reduces the integration of the conformal geodesic equations to quadratures. This completes the proof of Theorem 1.1.

Separating (4.20) with a constant $Q$ gives

$$
\dot{\eta}=\frac{4 \eta G_{\eta}}{\eta+\zeta+2 m}, \quad \dot{\zeta}=\frac{4 \zeta F_{\zeta}}{\eta+\zeta+2 m}
$$

with

$$
4 \eta G_{\eta}=U(\eta, E, Q), \quad 4 \zeta F_{\zeta}=U(\zeta,-E,-Q)
$$

where

$$
\begin{aligned}
U(x, E, Q) & =\sqrt{u_{0}+u_{1} x+u_{2} x^{2}+u_{3} x^{3}+u_{4} x^{4}}, \\
u_{0} & =-4(J+E m)^{2}, \quad u_{1}=4\left(Q+m\left(\mu^{2}+2 J e-2 E^{2}\right)\right), \\
u_{2} & =4\left(\mu^{2}-m^{2} e^{2}-E^{2}+J e+3 E m e\right), \quad u_{3}=4 e(E-m e), \quad u_{4}=-e^{2} .
\end{aligned}
$$

Therefore (4.21) imples that the unparametrised conformal geodesic equations are solvable in terms of elliptic functions:

$$
\int \frac{d \eta}{U(\eta, E, Q)}=\int \frac{d \zeta}{U(\zeta,-E,-Q)}
$$

\section{Circles on Eguchi-Hanson}

The Eguchi-Hanson (EH) metric [11]

$$
g=f^{-2} d r^{2}+\frac{1}{4} r^{2}\left(\sigma_{1}^{2}+\sigma_{2}^{2}\right)+\frac{1}{4} r^{2} f^{2} \sigma_{3}^{2}, \quad \text { where } \quad f=\sqrt{1-\frac{\alpha^{4}}{r^{4}}}, \quad \alpha=\text { const }
$$

is another special case of $(3 \cdot 12)$. It corresponds to

$$
V=\frac{1}{r_{1}}+\frac{1}{r_{2}}
$$

where $r_{1}, r_{2}$ are Euclidean distances from points $P_{1}, P_{2}$ which we can chose to be

$$
P_{1}=(0,0,-\alpha), \quad P_{2}=(0,0, \alpha)
$$

for constant positive $\alpha$. To achieve regularity the period of the $\psi$ coordinate in $\sigma_{3}$ should be $2 \pi$ rather than $4 \pi$. Therefore the surfaces of constant $r$ are real projective spaces, and at large $r$ the metric looks like $\mathbb{R}^{4} / \mathbb{Z}_{2}$ rather than Euclidean space. The Eguchi-Hanson is an example of an asymptotically locally Euclidean (ALE) manifold (see e.g. [7, Chapter 9]).

The isometry group of $\mathrm{EH}$ is enhanced to $S O(3) \times U(1)$, but this time it is the $S O(3)$ which acts tri-holomorphically (so that EH can be put in the form (3.12) in many ways). The $U(1)$ action is not tri-holomorphic. The parallel basis of $\Lambda^{2}$ is given by

$$
\Omega^{i}=e^{i} \wedge e^{4}+\frac{1}{2} \varepsilon^{i j k} e^{j} \wedge e^{k}
$$

with

$$
e^{1}=\frac{1}{2} r \sigma_{1}, \quad e^{2}=\frac{1}{2} r \sigma_{2}, \quad e^{3}=\frac{1}{2} r f \sigma_{3}, \quad e^{4}=f^{-1} d r
$$


PROPOSITION 5.1. All conformal geodesics on the SO(3) orbits of constant $r$ in the Eguchi-Hanson manifold are of the form

$$
\begin{aligned}
& \left(u^{1}\right)^{2}+\left(u^{2}\right)^{2}+\left(u^{3}\right)^{2}=1, \quad c_{1} u^{1}+c_{2} u^{2}=\frac{r f}{2\left(1-f^{2}\right)}\left(h^{2}-2\left(c_{1}^{2}+c_{2}^{2}\right)-2 m_{1}\right), \\
& \text { where } h=\frac{1-f^{2}}{f r} u^{3}+\frac{m_{0}}{c_{1}^{2}+c_{2}^{2}}
\end{aligned}
$$

and $\left(u^{1}, u^{2}, u^{3}\right)$ depend on $(\phi, \psi, \theta)$ as in (5.31) where $\left(c_{1}, c_{2}, c_{3}, m_{1}, m_{2}, p_{1}, p_{2}, p_{3}\right)$ are constants of integration.

We shall first perform the general analysis of the circle equations using the Lorentz force system (3.7), and reduce the equations to a system of three 1st order ODEs (5.32a) for three unknown functions. We shall then show that this system is completely solvable under the additional assumption $r=$ const. This will give the proof of Proposition 5.1.

With $a$ determined in terms of $u$ by (3.9) (note that now the components of $a$ and $u$ refer to the basis (5.24)) the circle equations reduce to the definition of the acceleration $a=\nabla_{u} u$, which becomes

$$
\begin{aligned}
& \dot{u}^{1}=2 \frac{k^{4}}{r \sqrt{1-k^{4}}} u^{2} u^{3}-\frac{\sqrt{1-k^{4}}}{r} u^{1} u^{4}+c^{2} u^{3}-c^{3} u^{2}-c^{1} u^{4}, \\
& \dot{u}^{2}=-2 \frac{k^{4}}{r \sqrt{1-k^{4}}} u^{1} u^{3}-\frac{\sqrt{1-k^{4}}}{r} u^{2} u^{4}+c^{3} u^{1}-c^{1} u^{3}-c^{2} u^{4}, \\
& \dot{u}^{3}=-\frac{1+k^{4}}{r \sqrt{1-k^{4}}} u^{3} u^{4}+c^{1} u^{2}-c^{2} u^{1}-c^{3} u^{4}, \\
& \dot{u}^{4}=\frac{\sqrt{1-k^{4}}}{r}\left(1-\left(u^{4}\right)^{2}\right)+2 \frac{k^{4}}{r \sqrt{1-k^{4}}}\left(u^{3}\right)^{2}+c^{1} u^{1}+c^{2} u^{2}+c^{3} u^{3},
\end{aligned}
$$

where $k \equiv \alpha / r$. In this case we can also find the potentials of the triple of parallel two-forms

$$
\begin{aligned}
& \Omega^{1}=e^{1} \wedge e^{4}+e^{2} \wedge e^{3}=d\left(-\frac{r f}{2} e^{1}\right) \\
& \Omega^{2}=e^{2} \wedge e^{4}+e^{3} \wedge e^{1}=d\left(-\frac{r f}{2} e^{2}\right) \\
& \Omega^{3}=e^{3} \wedge e^{4}+e^{1} \wedge e^{2}=d\left(-\frac{r}{2 f} e^{3}\right) .
\end{aligned}
$$

The relation

$$
u=\dot{\phi} \frac{\partial}{\partial \phi}+\dot{\theta} \frac{\partial}{\partial \theta}+\dot{\psi} \frac{\partial}{\partial \psi}+\dot{r} \frac{\partial}{\partial r}=u^{a} E_{a}
$$

gives

$$
\begin{aligned}
& u^{1}=\frac{1}{2} r(\sin (\theta) \sin (\psi) \dot{\phi}+\cos (\psi) \dot{\theta}) \\
& u^{2}=\frac{1}{2} r(\sin (\theta) \cos (\psi)(\dot{\phi})-\sin (\psi) \dot{\theta})
\end{aligned}
$$




$$
\begin{aligned}
& u^{3}=\frac{1}{2} r f(\dot{\psi}+\cos (\theta) \dot{\phi}) \\
& u^{4}=\frac{1}{f} \dot{r} .
\end{aligned}
$$

\section{5·1. Magnetic first integrals and right invariant vector fields}

The equations (5.26) correspond to a forced geodesic motion of a charged particle in a constant self-dual Maxwell field $F=c^{1} \Omega^{1}+c^{2} \Omega^{2}+c^{3} \Omega^{3}$

$$
u^{b} \nabla_{b} u^{a}=F^{a}{ }_{b} u^{b} .
$$

Let $\Phi$ be a potential for $F=d \Phi$. Using (5.27) we find

$$
\Phi=-\frac{1}{2}\left(c_{1} r f e^{1}+c_{2} r f e^{2}+c_{3} \frac{r}{f} e^{3}\right) .
$$

For any tri-holomrphic Killing vector $R$, such that $\mathcal{L}_{R} \Phi=0$ (this can always be arranged by adding a total derivative of a function to $\Phi$ ), the function

$$
p \equiv R^{a}\left(u_{a}+\kappa \Phi_{a}\right)
$$

is a first integral of (5.29) (here $\kappa$ is a numerical factor and the value of $\kappa$ can be absorbed into constants $c^{i}$ ).

Given the left-invariant one-forms $\sigma_{i}$, let $L_{i}$ be the dual basis of left-invariant vector fields, i. e. $\left.L_{i}\right\lrcorner \sigma_{j}=\delta_{i j}$, and

$$
\left[L_{i}, L_{j}\right]=\frac{1}{2} \epsilon_{i j k} L_{k} .
$$

The tri-holomorphic and isometric $S O(3)$ action is generated by the right-invariant vector fields $R_{j}$ on $S O(3)$ such that

$$
\left[L_{i}, R_{j}\right]=0, \quad\left[R_{i}, R_{j}\right]=-\frac{1}{2} \epsilon_{i j k} R_{k} .
$$

These vector fields can be expanded in terms of $L_{i}$ s and therefore also in terms of the dual basis $E_{1}, E_{2}, E_{3}$ as

$$
R_{i}=M^{j}{ }_{i} E_{j},
$$

where the components of the matric $M$ are explicit functions of $(r, \psi, \theta, \phi)$, and we have verified that $\mathcal{L}_{R_{i}} \Phi=0$. Combining this with (5.30) leads to three first integrals

$$
p_{i} \equiv R_{i}{ }^{a}\left(u_{a}+\kappa \Phi_{a}\right)=M^{j}{ }_{i}\left(u_{j}+\kappa \Phi_{j}\right) .
$$

These are three linear equations for $\mathbf{u}$ which can be soved as $\mathbf{u}=M^{-1} \mathbf{p}-\kappa \Phi$, or (using the explicit form of $M$ ) 


$$
\begin{aligned}
u^{1} & =\frac{\kappa}{2} c_{1} r f+\frac{1}{r}\left(p_{2}(\cos \phi \cos \psi-\sin \phi \sin \psi \cos \theta)\right. \\
& \left.-p_{3}(\sin \phi \cos \psi-\cos \phi \sin \psi \cos \theta)+p_{1} \sin \theta \sin \psi\right) \\
u^{2} & =\frac{\kappa}{2} c_{2} r f+\frac{1}{r}\left(-p_{2}(\cos \phi \sin \psi-\sin \phi \cos \psi \cos \theta)\right. \\
& \left.+p_{3}(\sin \phi \sin \psi-\cos \phi \cos \psi \cos \theta)+p_{1} \sin \theta \cos \psi\right) \\
u^{3} & =\frac{\kappa}{2 f} c_{3} r+\frac{1}{r f}\left(p_{1} \cos \theta+p_{2} \sin \phi \sin \theta+p_{3} \cos \phi \sin \theta\right) .
\end{aligned}
$$

\subsection{A final system of 1 st order ODEs}

Comparing the expressions $(5 \cdot 31)$ with $(5 \cdot 28)$ gives a system of three 1 st order equations for three unknown functions $\theta(s), \psi(s), \phi(s)$

$$
\begin{aligned}
\dot{\phi}= & \frac{1}{r^{2}}\left(2 p_{1}-2 \cot \theta\left(p_{2} \sin \phi+p_{3} \cos \phi\right)\right)+\kappa f \frac{c_{1} \sin \psi+c_{2} \cos \psi}{\sin \theta} \\
\dot{\theta}= & \frac{1}{r^{2}}\left(p_{2} \cos \phi-p_{3} \sin \phi\right)+\kappa f\left(c_{1} \cos \psi-c_{2} \sin \psi\right) \\
\dot{\psi}= & \left.\kappa\left(f \cot \theta\left(c_{1} \sin \psi+c_{2} \cos \psi\right)+\frac{1}{f^{2}} c_{3}\right)\right)+\frac{2}{r^{2} f^{2} \sin \theta}\left(p_{2} \sin \phi+p_{3} \cos \phi\right) \\
& +\frac{2 \alpha^{4}}{r^{6} f^{2} \sin \theta}\left(\cos \theta^{2}\left(p_{2} \sin \phi+p_{3} \cos \phi\right)-p_{1} \cos \theta \sin \theta\right)
\end{aligned}
$$

The equation for $\dot{r}$ can now be obtained from the 1st integral $g(u, u)=1$.

\subsection{An integrable case: circles on $S O(3)$ orbits}

In this section we shall establish the complete integrability of the of the conformal geodesic equations under additional assumption that the conformal geodesics lie on surfaces of constant $r$.

Proof of Proposition 5.1. The circle equations can be reduced to a quadrature under the additional assumption that $\dot{r}=0$, that is the circles are confined to the orbits of the isometric $S O(3)$ action which are surfaces of constant $r$. The condition $\dot{r}=0$ is equivalent to $u^{4}=0$. Set

$$
u^{1}=\alpha(s), \quad u^{2}=\beta(s), \quad u^{3}=\gamma(s), \quad \frac{k^{4}}{r \sqrt{1-k^{4}}}=R=\text { const. }
$$

Equations (5.26) take the form

$$
\begin{aligned}
& \dot{\alpha}=R \beta \gamma+c_{2} \gamma-c_{3} \beta \\
& \dot{\beta}=-R \alpha \gamma+c_{3} \alpha-c_{1} \gamma \\
& \dot{\gamma}=c_{1} \beta-c_{2} \alpha .
\end{aligned}
$$

Algebraic manipulations with these equations give expressions for $\alpha$ and $\beta$ in terms of $\gamma$ and its derivatives 


$$
\begin{aligned}
& \alpha=-\frac{c_{1}}{\left(c_{1}^{2}+c_{2}^{2}\right)\left(R \gamma-c_{3}\right)} \ddot{\gamma}-\frac{c_{2}}{c_{1}^{2}+c_{2}^{2}} \dot{\gamma}-\frac{c_{1}}{R \gamma-c_{3}} \gamma \\
& \beta=-\frac{c_{2}}{\left(c_{1}^{2}+c_{2}^{2}\right)\left(R \gamma-c_{3}\right)} \ddot{\gamma}+\frac{c_{1}}{c_{1}^{2}+c_{2}^{2}} \dot{\gamma}-\frac{c_{2}}{R \gamma-c_{3}} \gamma .
\end{aligned}
$$

The last equation in (5.33) now holds identically. The first two equations reduce to a single 3rd ODE

$$
h \dddot{h}=\left(\ddot{h}-h^{3}+m_{0}\right) \dot{h}, \quad \text { where } h(s)=R \gamma(s)-c_{3}, \quad \text { and } m_{0}=c_{3}\left(c_{1}^{2}+c_{2}^{2}\right) .
$$

This equation can be integrated in terms of elliptic functions

$$
s-m_{3}=2 \int \frac{d h}{\sqrt{-h^{4}+4 m_{1} h^{2}-8 m_{0} h+4 m_{2}}},
$$

where $m_{0}, m_{1}, m_{2}, m_{3}$ are constants of integration. Inverting this formula for $h$, and substituting in the expressions above gives $\alpha(s), \beta(s)$. The elliptic functions are however not neccesary to describe the conformal circles. To see this differentiate (5.36) twice, to obtain expressions for $\dot{h}$ and $\ddot{h}$ in terms of $h$. These expressions are then substituted into (5.34), and (remembering that $\gamma=R^{-1}\left(h(s)+c_{3}\right)$ ) yield the explicit parametrisation $\mathbf{u}(h)$ of $u^{1}, u^{2}, u^{3}$ in terms of a parameter $h$, and five integration constant (note that there is one constraint on the constants $m_{0}, m_{1}, m_{2}, m_{3}, c_{1}, c_{2}$ coming from $\left.g(u, u)=1\right)$. The formulae for $\mathbf{u}(h)$ are now substituted into (5.31) which yields relations of the form

$$
G_{1}(\phi, \psi, \theta, h)=0, \quad G_{2}(\phi, \psi, \theta, h)=0, \quad G_{3}(\phi, \psi, \theta, h)=0,
$$

where the functions $G_{i}$ can be read-off from (5.31), and depend on the 8 constants of integrations (five from the procedure above, and $p_{1}, p_{2}, p_{3}$ ). The parameter $h$ may be elliminated between these three relations which gives unparametrised conformal circles on the $r=$ const orbits in the Eguchi-Hanson space. They are given by $(5 \cdot 25)$.

\subsection{Separability of the Hamilton-Jacobi equation}

In this section we shall find another integrable subcase of the conformal geodesic equations on the Eguchi-Hanson background. This will be done by separating the associated Hamilton-Jacobi equation. Following [15] again, we introduce prolate spheroidal coordinates adapted to the potential $(5 \cdot 23)$

$$
\zeta=\frac{1}{2 \alpha}\left(r_{1}+r_{2}\right), \quad \lambda=\frac{1}{2 \alpha}\left(r_{1}-r_{2}\right)
$$

so these are constant on confocal ellipses and confocal hyperbolae respectively. Introduce the angles $\theta_{1}, \theta_{2}$ as the angles supported at $p_{1}, p_{2}$ respectively w.r.t. the $z$-axis; now

$$
r_{1}=\alpha(\zeta+\lambda), r_{2}=\alpha(\zeta-\lambda), \quad \cos \theta_{1}=\frac{\zeta \lambda+1}{\zeta+\lambda}, \cos \theta_{2}=\frac{\zeta \lambda-1}{\zeta-\lambda} .
$$

We need $V$ and $\omega$ in these coordinates: for the Eguchi-Hanson metric

$$
V=\frac{1}{r_{1}}+\frac{1}{r_{2}}=\frac{2 \zeta}{\zeta^{2}-\lambda^{2}}, \omega=\Omega d \phi \quad \text { with } \quad \Omega=-2 \frac{\lambda\left(\zeta^{2}-1\right)}{\zeta^{2}-\lambda^{2}},
$$


with conventions

$$
\alpha\left(\zeta^{2}-1\right) V_{\zeta}=\Omega_{\lambda}, \quad-\alpha\left(1-\lambda^{2}\right) V_{\lambda}=\Omega_{\zeta}
$$

Claim

$$
d \rho^{2}+d z^{2}+\rho^{2} d \phi^{2}=\alpha^{2}\left(\zeta^{2}-\lambda^{2}\right)\left(\frac{d \zeta^{2}}{\zeta^{2}-1}+\frac{d \lambda^{2}}{1-\lambda^{2}}\right)+\alpha^{2}\left(\zeta^{2}-1\right)\left(1-\lambda^{2}\right) d \phi^{2}
$$

whence

$g_{E H}=V\left(\alpha^{2}\left(\zeta^{2}-\lambda^{2}\right)\left(\frac{d \zeta^{2}}{\zeta^{2}-1}+\frac{d \lambda^{2}}{1-\lambda^{2}}\right)+\alpha^{2}\left(\zeta^{2}-1\right)\left(1-\lambda^{2}\right) d \phi^{2}\right)+V^{-1}(d \psi+\Omega d \phi)^{2}$.

From the geodesic equations we obtain constants of the motion

$$
p_{\psi}=V^{-1}(\dot{\psi}+\Omega \dot{\phi}):=E, \quad p_{\phi}=\alpha^{2} V\left(\zeta^{2}-1\right)\left(1-\lambda^{2}\right) \dot{\phi}+\Omega E:=J,
$$

from the Killing vectors $K:=\partial_{\psi}$ and $L:=\partial_{\phi}$, so that

$$
p_{\phi}-\Omega p_{\psi}=\alpha^{2} V\left(\zeta^{2}-1\right)\left(1-\lambda^{2}\right) \dot{\phi}
$$

Seek a solution

$$
S=E \psi+J \phi+F(\zeta)+G(\lambda)
$$

for the Hamilton-Jacobi equation then

$$
\mu^{2}=\frac{\zeta^{2}-1}{\alpha^{2} V\left(\zeta^{2}-\lambda^{2}\right)} F_{\zeta}^{2}+\frac{1-\lambda^{2}}{\alpha^{2} V\left(\zeta^{2}-\lambda^{2}\right)} G_{\lambda}^{2}+\frac{(J-\Omega E)^{2}}{\alpha^{2} V\left(\zeta^{2}-1\right)\left(1-\lambda^{2}\right)}+V E^{2} .
$$

Multiply by $\alpha^{2} V\left(\zeta^{2}-\lambda^{2}\right)$ and simplify to obtain

$$
2 \alpha \zeta=\left(\zeta^{2}-1\right) F_{\zeta}^{2}+\left(1-\lambda^{2}\right) G_{\lambda}^{2}+J^{2}\left(\frac{1}{\zeta^{2}-1}+\frac{1}{1-\lambda^{2}}\right)+\frac{4 J E \lambda}{1-\lambda^{2}}+\frac{4 E^{2}}{1-\lambda^{2}},
$$

which separates.

Up to this point we are following [15]. To extend to the Lorentz force law, we need a Maxwell potential for $F_{a b}$ which commutes with $K$ and $L$. Now there is a problem: for antiself-dual Taub-NUT there was no loss in generality in choosing $F=-\Omega^{3}$ but in EguchiHanson there is, as $K$ will Lie-drag any constant $\phi$ but $L$ will not. Thus we can obtain integrability for a sub-class of conformal geodesics, those for which $F=-\Omega^{3}$, but not for all. With this restriction then we have

$$
\Omega^{3}=-(d \psi+\omega) \wedge d z+V d x \wedge d y .
$$

If the potential for $-\Omega_{3}$ is

$$
\Phi=-z(d \psi+\omega)+X d \phi=-z(d \psi+\Omega d \phi)+X d \phi,
$$

then

$$
d X \wedge d \phi=z d \Omega \wedge d \phi-V \rho d \rho \wedge d \phi
$$

This can be solved by

$$
X=z \Omega+2 \alpha \zeta
$$


when

$$
\Phi=(X-z \Omega) d \phi-z d \psi=2 \alpha \zeta d \phi-\alpha \zeta \lambda d \psi .
$$

To modify the Hamilton-Jacobi equation we make the replacements

$$
J \rightarrow J+2 e \alpha \zeta, \quad E \rightarrow E-e \alpha \zeta \lambda
$$

into $(5 \cdot 37)$. The added terms are

$$
4 e \alpha J \frac{\zeta^{3}}{\zeta^{2}-1}+4 e^{2} \alpha^{2} \frac{\zeta^{4}}{\zeta^{2}-1}
$$

so this still separates, and this sub-class of the conformal geodesic equations is completely integrable.

\section{Circles on $\mathbb{C P}^{2}$}

Consider the Fubini-Study metric on $\mathbb{C P}^{2}$. It is Einstein with the Ricci scalar equal to 24, has anti-self-dual (ASD) Weyl tensor, and is Kähler, but with the ASD Kähler two-form (this is some times referred to as 'opposite orientation'). The local form of the metric is (see e.g. [14])

$$
g_{\mathbb{C P}^{2}}=\frac{d r^{2}}{\left(1+r^{2}\right)^{2}}+\frac{1}{4} \frac{r^{2} \sigma_{3}^{2}}{\left(1+r^{2}\right)^{2}}+\frac{1}{4} \frac{r^{2}}{1+r^{2}}\left(\sigma_{1}^{2}+\sigma_{2}^{2}\right) .
$$

The metric is regular everywhere on $\mathbb{C P}^{2}$, and the apparent singularity at $r=0$ results from using spherical polar coordinates. The metric is also regular at $r=\infty$. To see this set $r=$ $\rho^{-1}$. Fixing $(\phi, \theta)$ now gives $g \sim d \rho^{2}+(1 / 4) \rho^{2} d \psi^{2}$ near $\rho=0$. This is a removable bolt singularity (see [7]) if $\psi$ is periodic with the period $2 \pi$. At $\rho=0$ the three-dimensional orbits of $S U$ (2) collapse to a two-sphere of constant radius. We shall refer to this as the $\mathbb{C P}^{1}$ at infinity.

It was shown in [9] that in case of ASD Einstein manifolds with non-zero Ricci scalar there is a one-to-one correspondence between Killing vectors, and self-dual CKY tensors: If $K$ is a Killing vector, then its self dual derivative

$$
Y \equiv \frac{1}{2}(d K+* d K)
$$

satisfies (2.5). The Fubini-Study metric is a symmetric space $M=S U(3) / U(2)$, so it has 8 Killing vectors generating the Lie algebra $\mathfrak{s u}(3)$, and therefore 8 self-dual CKYs. This gives rise to 8 first integrals of the form (2.6). The 9th first integral is given by the parallel CKY which is the anti-self-dual Kähler form. In Section we aim to use these first integrals to find all conformal geodesics on $\mathbb{C P}^{2}$. We shall first prove.

Proposition 6.1. All conformal geodesics on the Fubini-Study metric (6.38) on $\mathbb{C P}^{2}$ on the surfaces of constant $r$ are trajectories of the Killing vector $\partial / \partial \psi$, or are of the form $\chi=\operatorname{arccot}\left(\frac{\kappa\left(c_{2} \cos (\kappa s)-\sin (\kappa s)\right)}{Q\left(c_{3}+c_{2} \sin (\kappa s)+\cos (\kappa s)\right)}\right), \quad \theta=\operatorname{arccot}\left(\frac{Q-\dot{\chi}}{P \sin \chi}\right), \quad \phi=P \int \frac{\sin \chi}{\sin \theta} d s$, 
where

$$
Q=2 \gamma / r-\gamma r, \quad P=\frac{2 \sqrt{\left(1+r^{2}\right)\left(1-\gamma^{2}\right)}}{r}, \quad \kappa=\sqrt{P^{2}+Q^{2}}
$$

and $r, \gamma, c_{1}, c_{2}, c_{3}$ are constants.

We then deduce the the general form of conformal geodesics:

THEOREM 6.2. All conformal geodesics of the Fubini-Study metric on $\mathbb{C P}^{2}$ are of the form $\rho \circ \Gamma$ where $\Gamma$ is some conformal geodesic on the $r=$ const surface given in Proposition $6 \cdot 1$, and $\rho$ is an isometry of the Fubini-Study metric.

Proof of Proposition 6·1. Pick a tetrad of one-forms

$$
e^{1}=\frac{r}{2 \sqrt{1+r^{2}}} \sigma_{1}, \quad e^{2}=\frac{r}{2 \sqrt{1+r^{2}}} \sigma_{2}, \quad e^{3}=\frac{r}{2\left(1+r^{2}\right)} \sigma_{3}, \quad e^{4}=\frac{d r}{1+r^{2}},
$$

so that the metric $(6 \cdot 38)$ is $g=\delta_{a b} e^{a} e^{b}$, and set

$$
u=\alpha E_{1}+\beta E_{2}+\gamma E_{3}+\delta E_{4}, \quad a=a^{1} E_{1}+a^{2} E_{2}+a^{3} E_{3}+a^{4} E_{4},
$$

where $E_{a}, a=1,2,3,4$ is the dual tetrad of vector fields, and $|u|^{2}=1$. The conformal geodesic equations $(2.4)$ become

$$
\begin{aligned}
& a^{1}=\dot{\alpha}+2 r \beta \gamma+\frac{1}{r} \alpha \delta \\
& a^{2}=\dot{\beta}-2 r \gamma \alpha+\frac{1}{r} \beta \delta \\
& a^{3}=\dot{\gamma}-r \gamma \delta+\frac{1}{r} \gamma \delta \\
& a^{4}=\dot{\delta}+r \gamma^{2}-\frac{1}{r}\left(1-\delta^{2}\right)
\end{aligned}
$$

and

$$
\begin{aligned}
& \dot{a}^{1}=\frac{1}{r}\left(\beta a^{3}-\alpha a^{4}-\gamma a^{2}\right)-2 r \gamma a^{2}-|a|^{2} \alpha \\
& \dot{a}^{2}=\frac{1}{r}\left(\gamma a^{1}-\beta a^{4}-\alpha a^{3}\right)+2 r \gamma a^{1}-|a|^{2} \beta \\
& \dot{a}^{3}=\frac{1}{r}\left(\alpha a^{2}-\gamma a^{4}-\beta a^{1}\right)+r \gamma a^{4}-|a|^{2} \gamma \\
& \dot{a}^{4}=\frac{1}{r}\left(\alpha a^{1}+\beta a^{2}+\gamma a^{3}\right)-r \gamma a^{3}-|a|^{2} \delta,
\end{aligned}
$$

together with

$$
|u|^{2}=1, \quad \delta_{e f} a^{e} a^{f}=|a|^{2}, \quad \text { where } u=(\alpha, \beta, \gamma, \delta) .
$$

Note that $g(u, a)=0$ is satisfied identically if $u^{a}$ is unit. 
The CKY corresponding to the Killing vector $\partial / \partial \psi$ is

$$
\frac{r^{2}}{r^{2}+1}\left(e^{1} \wedge e^{2}+e^{3} \wedge e^{4}\right)
$$

and gives rise to a first integral

$$
C_{Y}:=\frac{r^{2}}{r^{2}+1}\left(\alpha a^{2}-\beta a^{1}+\gamma a^{4}-\delta a^{3}\right)+\frac{2 r}{r^{2}+1} \gamma .
$$

The ASD Kähler form is

$$
e^{1} \wedge e^{2}-e^{3} \wedge e^{4}
$$

This gives rise to a first integral

$$
C_{K}:=\alpha a^{2}-\beta a^{1}-\gamma a^{4}+\delta a^{3} .
$$

Combining the two gives

$$
\begin{aligned}
& \left(r^{2}+1\right) C_{Y}-r^{2} C_{K}=2 r^{3} \gamma\left(\gamma^{2}+\delta^{2}\right)+2 r^{2}(\gamma \dot{\delta}-\delta \dot{\gamma}) . \\
& \left(r^{2}+1\right) C_{Y}+r^{2} C_{K}=4 r^{3} \gamma\left(\gamma^{2}+\delta^{2}\right)+2 r^{2}(\alpha \dot{\beta}-\beta \dot{\alpha})+2 r \gamma-4 r^{3} \gamma .
\end{aligned}
$$

The first integral $|a|^{2}$ after some algebra simplifies to

$$
\begin{aligned}
|a|^{2}= & \delta_{e f} \dot{u}^{e} \dot{u}^{f}+r^{2} \gamma^{2}\left(4-3\left(\gamma^{2}+\delta^{2}\right)\right)+2 r \gamma(2 \beta \dot{\alpha}-2 \alpha \dot{\beta}+\gamma \dot{\delta}-\delta \dot{\gamma}) \\
& -2 \gamma^{2}-\frac{2}{r} \dot{\delta}+\frac{1}{r^{2}}\left(1-\delta^{2}\right) .
\end{aligned}
$$

We now aim to find all circles such that $\delta=0$, or equivalently all circles lying on surfaces of constant $r$. The vector field $u$ is unit, so set $\beta=\sqrt{1-\alpha^{2}-\gamma^{2}}$. Equations (6.46a, 6.46b) and (6.47) give

$$
\begin{aligned}
& 0=2(r \gamma)^{3}-r^{2}\left(C_{Y}-C_{K}\right)-C_{Y} \\
& 0=2 r^{2}(\alpha \dot{\beta}-\beta \dot{\alpha})+4(r \gamma)^{3}-4 r^{3} \gamma+2 r \gamma-r^{2}\left(C_{K}+C_{Y}\right)-C_{Y} \\
& A=\dot{\alpha}^{2}+\dot{\beta}^{2}+(r \gamma)^{2}\left(4-3 \gamma^{2}\right)+4 r \gamma(\beta \dot{\alpha}-\alpha \dot{\beta})-2 \gamma^{2}+\frac{1}{r^{2}}
\end{aligned}
$$

so that $\gamma$ is a constant along circles (and this constant depends on $r$ ). We also get $a^{4}=$ $r \gamma^{2}-1 / r$, which is a constant along circles, and $a^{3}=0$. Substituting $\beta=\sqrt{1-\alpha^{2}-\gamma^{2}}$ into (6.48b) yields a 1 st order equation for $\alpha(s)$ which we can integrate to find

$$
\begin{aligned}
\alpha(s) & =\sqrt{1-\gamma^{2}} \sin \left(B s+c_{1}\right), \quad \beta(s)=\sqrt{1-\gamma^{2}} \cos \left(B s+c_{1}\right), \quad \text { where } \\
B & =\frac{4 r^{3}\left(\gamma^{3}-\gamma\right)-r^{2}\left(C_{K}+C_{Y}\right)+2 r \gamma-C_{Y}}{2 r^{2}\left(1-\gamma^{2}\right)}, \quad \text { where } \gamma \neq \pm 1
\end{aligned}
$$

and $\gamma$ is a root of (6.48a).

To find any additional constraints on $B$ we substitute $u=(\alpha(s), \beta(s), \gamma, 0)$ into the conformal geodesic equations. We find that these equations hold iff

$$
B=-3 \gamma r, \quad \text { or } \quad \gamma^{2} r^{2}+\gamma B+1=0 .
$$


The squared norm of acceleration (6.48c) is constant along conformal circles, and is given by

$$
|a|^{2}=B^{2}\left(1-\gamma^{2}\right)+4 r \gamma B\left(1-\gamma^{2}\right)+r^{2} \gamma^{2}\left(4-3 \gamma^{2}\right)-2 \gamma^{2}+\frac{1}{r^{2}} .
$$

We can now compute the complex torsion $\tau=C_{K} /|a|$ and find that it can take any value between $(-1,1)$ if $B=-3 \gamma r$, but $\tau^{2}=1$ if $B=-\gamma r^{2}-\gamma^{-1}$. We shall therefore take $B=$ $-3 \gamma r$ from now on, and return to this point in the proof of Theorem 6.2.

If $\gamma= \pm 1$, then the circle is a trajectory of the Killing vector $\partial / \partial \psi=r /\left(r^{2}+1\right) E_{3}$, and $\alpha=\beta=0$ with the corresponding acceleration $a=\left(r^{2}-1\right) / r E_{4}$. In this case we find that $u=E_{3}$ and

$$
\nabla_{u} \nabla_{u} u=-\frac{\left(r^{2}-1\right)^{2}}{r^{2}} u
$$

so the circle equations (2.4) hold ${ }^{3}$ with $|a|^{2}=\left(r^{2}-1\right)^{2} / r^{2}$.

To complete the calculation for $\gamma \neq \pm 1$ we need to find the dependence of the coordinates $(\phi, \theta, \psi, r)$ on the arc-lengh parameter $s$ along the circles (6.49). Setting

$$
u=\dot{\phi} \frac{\partial}{\partial \phi}+\dot{\theta} \frac{\partial}{\partial \theta}+\dot{\psi} \frac{\partial}{\partial \psi}+\dot{r} \frac{\partial}{\partial r},
$$

equating it to $u$ in (6.40) and substituting (6.49) yields a coupled system of three 1st order ODEs

$$
\begin{aligned}
\dot{\phi} & =2 \frac{\sqrt{1+r^{2}}}{r} \frac{\sqrt{1-\gamma^{2}}}{\sin \theta} \sin \left(\psi+B s+c_{1}\right), \\
\dot{\theta} & =2 \frac{\sqrt{1+r^{2}}}{r} \sqrt{1-\gamma^{2}} \cos \left(\psi+B s+c_{1}\right), \\
\dot{\psi} & =2 \frac{1+r^{2}}{r} \gamma-2 \frac{\sqrt{1+r^{2}}}{r} \sqrt{1-\gamma^{2}} \cot \theta \sin \left(\psi+B s+c_{1}\right), \\
\dot{r} & =0 .
\end{aligned}
$$

To simplify this slightly, set

$$
\chi=\psi+B s+c_{1}, \quad P=\frac{2 \sqrt{\left(1+r^{2}\right)\left(1-\gamma^{2}\right)}}{r}=\mathrm{const}, \quad Q=B+2 \frac{1+r^{2}}{r} \gamma=\mathrm{const}
$$

so that

$$
\dot{\phi}=P \frac{\sin \chi}{\sin \theta}, \quad \dot{\theta}=P \cos \chi, \quad \dot{\chi}=Q-P \cot \theta \sin \chi .
$$

We claim that this system is solvable by quadratures. Given $\chi(s)$ we find

$$
\theta=\operatorname{arccot}\left(\frac{Q-\dot{\chi}}{P \sin \chi}\right), \quad \text { and then } \quad \phi=P \int \frac{\sin \chi}{\sin \theta} d s .
$$

${ }^{3}$ We also note that in general $\dot{r}=\left(1+r^{2}\right) \delta$, and use this to verify that $u=E_{a}(a=1,2,3,4)$ satisfies the circle eq. In the last case we get $|a|^{2}=0$. 
The final equation for $\chi$ arises from the middle equation in (6.54):

$$
P^{2} \cos (\chi)^{3}-2 \cos (\chi) \dot{\chi}^{2}+3 Q \cos (\chi) \dot{\chi}-\cos (\chi)\left(P^{2}+Q^{2}\right)+\sin (\chi) \ddot{\chi}=0 .
$$

The general solution of this equation is given by

$$
\chi=\operatorname{arccot}\left(\frac{\kappa\left(c_{2} \cos (\kappa s)-\sin (\kappa s)\right)}{Q\left(c_{3}+c_{2} \sin (\kappa s)+\cos (\kappa s)\right)}\right), \quad \text { where } \quad \kappa=\sqrt{P^{2}+Q^{2}} .
$$

This can now be substituted in (6.55) to find the explicit form of $\theta$. It turns out that (according to MAPLE) even the $\phi$ integral can be computed in terms of elementary functions, but the answer is not illuminating. The integration will in any case introduce another constant call it $c_{4}$. Thus there exists a seven-dimensional family of conformal circles on surfaces of constant $r$. This family is parametrised by $c_{1}, c_{2}, c_{3}, c_{4}, C_{Y}, C_{K}, r$, and explicitly given by formulae $(6 \cdot 56,6.55,6.53)$, where $B$ is given by $(6.49)$, and $\gamma$ is given by $(6.48 \mathrm{a})$.

We shall now use the conformal geodesics on the $r=$ const surfaces and conjugate them with the elements of the isometry group $S U$ (3) to find all conformal geodesics on $\mathbb{C P}^{2}$. The fact that all conformal geodesics arise from this construction will follow from the results of [21] which we now recall.

We shall say that two circles $\Gamma_{1}$ and $\Gamma_{2}$ are congruent if there exists a holomorphic isometry (note - all isometries on $\mathbb{C P}^{2}$ are holomorphic) $\rho$ such that $\Gamma_{1}=\rho \circ \Gamma_{2}$.

In [21, Theorem 5.1] it has been shown that two circles on $\mathbb{C P}^{2}$ with the same complex torsion

$$
\tau=g_{\mathbb{C P}^{2}}(J(u), a /|a|)
$$

and curvature $|a|^{2}$ are congruent. Here $J$ is the complex structure of the ASD Kähler form on $\mathbb{C P}^{2}$ and we note that $\tau=C_{K} /|a|$, where $C_{K}$ is the first integral (6.45). We also note $\tau^{2} \leq|J(u)|^{2}|a|^{2} /|a|^{2}=1$, so that $\tau \in[-1,1]$. Doing the counting, at a point on $r=$ constant there are 2 dimensions of (unit) velocity $u$ and then 2 dimensions of acceleration orthogonal to velocity so with +3 for the dimension of $r=$ constant surface and -1 for the one-dimension of the curve that is 6 dimensions of conformal geodesics confined to $r=$ const. But there is a four-dimensional space of Killing vectors tangent to $r=$ constant to move them about, leaving 2 parameters. In the proof of Theorem 6.2 we shall show that these are $|a|$ and $\tau$.

The general counting for unparametrised conformal geodesics on $\mathbb{C P}^{2}$ is as follows: there are 3 degrees of freedom of velocity, 3 of acceleration, 4 of position minus 1 for the dimension of the curve. This gives gives a 9 dimensional space of conformal geodesics on $\mathbb{C P}^{2}$. There are 8 Killing vectors, but an orbit of $S U(3)$ has fixed $|a|$ and $\tau$ which is 7 dimensions in the space of conformal geodesics. Therefore there is always 1-dimensional isometry group stabilising a conformal geodesic i.e. every conformal geodesic $\Gamma(s)=\exp (s K) \Gamma(0)$, is a trajectory of some Killing vector $K$ which confirms the results of [20]. This counting is correct as long as the Killing vector $K$ is tangent to the conformal geodesic, rather than being vanishing on it. To examine this we look at fixed points of Killing vectors in $\mathbb{C P}^{2}$. A one parameter subgroup of the isometry group comes from an $S U$ (3) matrix, and fixed points correspond to eigenvectors; if the eigenvalues are distinct then the Killing vector vanishes at isolated points (like $\partial_{\phi}$ in the coordinate system $(6 \cdot 38)$ ) but if there is a repeat then the Killing vector vanishes on a $\mathbb{C P}^{1}$ in $\mathbb{C P}^{2}$ (like $\partial_{\psi}$ which vanishes at $r=\infty$ - the $\mathbb{C P}^{1}$ at infinity). So if a conformal geodesic does not lie on a $\mathbb{C P}^{1}$, then it can not lie in the zero set of a Killing so must be a Killing vector trajectory. If it does lie on a $\mathbb{C P}^{1}$ then there is a 
Killing vector that vanishes everywhere along the geodesic but it does not matter; take it to be the $\mathbb{C P}^{1}$ at infinity which is a metric sphere and extrinsically flat, so that the conformal geodesics are genuine circles and are Killing vector trajectories.

Proof of Theorem 6.2. In view of the discussion above, and the results of [21] it is sufficient to show that geodesics on constant $r$ surfaces from Proposition 6.1 can have arbitrary values of the torsion $\tau$, and the curvature $|a|^{2}$. We shall first consider the case when $\tau= \pm 1$. Then the trajectory of the Killing vector $\partial / \partial \psi$ is a conformal geodesic (6.51) with $|a|^{2}=\left(r^{2}-\right.$ $1)^{2} / r^{2}$ which can take any value in $[0, \infty)$.

We shall therefore assume that $\tau^{2}<1$, and show that for any value of $(\tau,|a|)$ there is a corresponding value of $(\gamma, r)$. This will be achieved by finding two relations between $(\tau,|a|)$ and $(\gamma, r)$.

Recall from (6.50) that $B=-3 \gamma r$, or $\gamma^{2} r^{2}+\gamma B+1=0$. In the latter case we find $C_{K}{ }^{2}=|a|^{2}$, so that $\tau= \pm 1$, but we already have a conformal geodesics with these values of $\tau$. We shall therefore assume that

$$
B=-3 \gamma r
$$

in which case we find

$$
|a|^{2}=\gamma^{2} r^{2}-2 \gamma^{2}+\frac{1}{r^{2}}
$$

and

$$
C_{K}=\frac{\gamma}{r}\left(1+r^{2}-2 \gamma^{2} r^{2}\right)
$$

As a check, calculate

$$
1-\tau^{2}=1-\frac{C_{K}^{2}}{|a|^{2}}=\frac{\left(1-\gamma^{2}\right)\left(1-2 r^{2} \gamma^{2}\right)^{2}}{\left(1-\gamma^{2}\right)+\gamma^{2}\left(r^{2}-1\right)^{2}}>0,
$$

as expected. Also (6.60) will give the sign of $C_{K} / \gamma$ (note that, given a conformal geodesic, then traversing it in the opposite direction switches the signs on $C_{K}$ and $\gamma$ ). Solving (6.59) for $\gamma^{2}$ gives

$$
\gamma^{2}=\frac{r^{2}|a|^{2}-1}{r^{2}\left(r^{2}-2\right)}
$$

(once we have $r^{2}$ we will need to check that this is less than 1). This relation together with

$$
C_{K}=-r^{3} \gamma^{3}+r \gamma\left(1+|a|^{2}\right)
$$

(which follows from (6.45)) gives a cubic

$$
F(X) \equiv(X A-1)(X-(2 A+1))^{2}-T A(X-2)^{3}=0, \quad \text { where } \quad X \equiv r^{2}, A \equiv|a|^{2}, T \equiv \tau^{2} .
$$

We will argue that this cubic has three positive roots which coalesce at $X=2$ when $A=1 / 2$.

Let us deal separately with $r^{2}=2$ : from (6.59) for this case $|a|^{2}=1 / r^{2}=1 / 2$ for any $\gamma^{2}$; then from $(6 \cdot 60)$

$$
\tau=\frac{C_{K}}{|a|}=\gamma\left(3-4 \gamma^{2}\right)
$$


and for any $\tau$ with $-1<\tau<1$, which is the currently allowed range, inspection of this cubic shows that there are three roots for $\gamma$ in $(-1,1)$.

Now we can suppose that $r^{2} \neq 2$. Substitute for $\gamma^{2}$ into the square of (6.60):

$$
\begin{gathered}
C_{K}^{2}=\frac{\gamma^{2}}{r^{2}}\left(1+r^{2}-2 \gamma^{2} r^{2}\right)^{2}=\frac{r^{2}|a|^{2}-1}{r^{4}\left(r^{2}-2\right)}\left(1+r^{2}-2 r^{2}\left(\frac{r^{2}|a|^{2}-1}{r^{2}\left(r^{2}-2\right)}\right)\right)^{2} \\
=\frac{\left(r^{2}|a|^{2}-1\right)}{\left(r^{2}-2\right)^{3}}\left(r^{2}-\left(1+2|a|^{2}\right)\right)^{2} .
\end{gathered}
$$

Rationalise:

$$
0=\left(r^{2}|a|^{2}-1\right)\left(r^{2}-\left(1+2|a|^{2}\right)\right)^{2}-C_{K}^{2}\left(r^{2}-2\right)^{3},
$$

and put $r^{2}=X$ to define the cubic

$$
\begin{aligned}
F(X):= & X^{3}\left(|a|^{2}-C_{K}^{2}\right)-X^{2}\left(1+2|a|^{2}+4|a|^{4}-6 C_{K}^{2}\right) \\
& +X\left(2+5|a|^{2}+4|a|^{4}+4|a|^{6}-12 C_{K}^{2}\right) \\
& -\left(1+4|a|^{2}+4|a|^{4}-8 C_{K}^{2}\right)
\end{aligned}
$$

whose vanishing we want. Note that, in $F$ :

(i) the coefficient of $X^{3}$ is

$$
|a|^{2}-C_{K}^{2}=\left(1-\tau^{2}\right)|a|^{2}
$$

which is strictly positive;

(ii) the coefficient of $X^{2}$ is

$$
-\left(1+2|a|^{2}\left(1-3 \tau^{2}\right)+4|a|^{4}\right)=-\left(\left(1-2|a|^{2}\right)^{2}+6|a|^{2}\left(1-\tau^{2}\right)\right)
$$

which is strictly negative;

(iii) the coefficient of $X$ is

$$
\begin{gathered}
2+5|a|^{2}+4|a|^{4}+4|a|^{6}-12 C_{K}^{2}>2-7|a|^{2}+4|a|^{4}+4|a|^{6} \\
=\left(2|a|^{2}-1\right)^{2}(|a|+2)
\end{gathered}
$$

where the first inequality uses $\tau^{2}<1$, so that this coefficient is strictly positive; (iv) and the constant term is

$$
-\left(1+4|a|^{2}+4|a|^{4}-8 C_{K}^{2}\right)=-\left(1-2|a|^{2}\right)^{2}-8|a|^{2}\left(1-\tau^{2}\right),
$$

which is strictly negative.

Thus $F(X)$ has no negative roots and at least one positive root. We will see below there are three positive roots, which coalesce at $r^{2}=2$ when $|a|^{2}=1 / 2$.

Next we note some particular values of $F$ :

$$
F\left(1 /|a|^{2}\right)=\frac{\tau^{2}}{|a|^{8}}\left(2|a|^{2}-1\right)^{3}, \quad F(2)=\left(2|a|^{2}-1\right)^{3}
$$


and

$$
F\left(1+2|a|^{2}\right)=-C_{K}^{2}\left(2|a|^{2}-1\right)^{3} .
$$

Now we can analyse the roots of $F$ :

(i) If $|a|^{2}=1 / 2$ then $r^{2}=2$ is a root, and by inspection of (6.62) we see that it is three times repeated. By the discussion above, there is now a value of $\gamma$ giving any value of $\tau$.

(ii) If $2|a|^{2}-1<0$ then the evaluations in (6.64) are both negative and that in (6.65) is positive, while

$$
1+2|a|^{2}<2<1 /|a|^{2}
$$

Therefore there is one root in each of the ranges $\left(0,1+2|a|^{2}\right),\left(1+2|a|^{2}, 2\right)$ and $\left(1 /|a|^{2}, \infty\right)$. All three make $\gamma^{2}$ in (6.61) positive but we shall choose the largest root $r_{3}^{2}$ which has

$$
r_{3}^{2}>1 /|a|^{2}>2
$$

so that there are positive $\delta, \epsilon$ with $\delta<\epsilon$ and

$$
r_{3}^{2}=1 /|a|^{2}+\delta=2+\epsilon .
$$

Now from (6.61)

$$
\gamma^{2}=\frac{r_{3}^{2}|a|^{2}-1}{r_{3}^{2}\left(r_{3}^{2}-2\right)}=\frac{\delta|a|^{2}}{\epsilon r_{3}^{2}}<\frac{\delta}{2 r_{3}^{2} \epsilon}<\frac{\delta}{4 \epsilon}<\frac{1}{4}<1,
$$

so that this choice of $r$ leads to a $\gamma$ in the allowed range.

(iii) if $2|a|^{2}-1>0$ then the evaluations in (6.64) are both positive and that in (6.65) is negative, while

$$
1 /|a|^{2}<2<1+2|a|^{2}
$$

Therefore there is one root in each of the ranges $\left(0,1 /|a|^{2}\right),\left(2,1+2|a|^{2}\right)$ and $(1+$ $\left.2|a|^{2}, \infty\right)$ and all make $\gamma^{2}$ in (6.61) positive. Again we choose the largest root $r_{3}^{2}$ which therefore has

$$
r_{3}^{2}>1+2|a|^{2}>2
$$

We choose positive $\delta, \epsilon$ with

$$
|a|^{2}=\frac{1}{2}+\epsilon, r_{3}^{2}=2+\delta
$$

so that also

$$
r_{3}^{2}=2+\delta>1+2|a|^{2}=2+2 \epsilon \text { i.e. } \delta>2 \epsilon .
$$


Now from $(6 \cdot 61)$

$$
\begin{aligned}
\gamma^{2} & =\frac{r_{3}^{2}|a|^{2}-1}{r_{3}^{2}\left(r_{3}^{2}-2\right)}=\frac{(1 / 2+\epsilon)(2+\delta)-1}{\delta(2+\delta)}=\frac{1}{4} \frac{\delta+4 \epsilon+2 \epsilon \delta}{\delta+\delta^{2} / 2} \\
& =\frac{1}{4}\left(1+\frac{4 \epsilon+2 \epsilon^{2}-(\delta-2 \epsilon)^{2} / 2}{\delta+\delta^{2} / 2}\right)<\frac{1}{4}\left(1+\frac{4 \epsilon+2 \epsilon^{2}}{\delta+\delta^{2} / 2}\right)
\end{aligned}
$$

and with $\delta>2 \epsilon$ :

$$
<\frac{1}{4}\left(1+\frac{4 \epsilon+2 \epsilon^{2}}{2 \epsilon+2 \epsilon^{2}}\right)<\frac{3}{4}<1,
$$

so again we have $\gamma$ in the allowed range.

We have shown that any allowed $\left(C_{K},|a|^{2}\right)$ can be obtained from an allowed $(r, \gamma)$.

\section{Remark.}

(i) A smooth curve $\Gamma$ parametrised by an arc-lengh is called a helix of proper order $d$, if there exist orthonormal vector fields $\left\{V_{1} \equiv \dot{\Gamma}, V_{2}, \ldots, V_{d}\right\}$ such that

$$
\nabla_{s} V_{j}=-\kappa_{j-1} V_{j-1}+\kappa_{j} V_{j+1}, \quad \text { where } j=1, \ldots, d
$$

and $V_{0}=V_{d} \equiv 0$, and the functions $\kappa_{j}=\kappa_{j}(s)$ (we set $\kappa_{0}=\kappa_{d} \equiv 0$ ) where $j=$ $1, \ldots, d-1$ are constant along $\Gamma$. Thus a helix of order 1 is a geodesic, and a helix of order 2 is a circle.

Let $\pi: S^{5} \rightarrow \mathbb{C P}^{2}$ be the Hopf fibration such that the round metric on $S^{5}$ is of the form

$$
g_{5}=(d t+\Theta)^{2}+g_{\mathbb{C P}^{2}},
$$

where $d \Theta=\Omega$ is the Kähler form for $\mathbb{C P}^{2}$ (this is also the derivative of $K=\partial / \partial t$ Killing vector). In [2] it is argued that horizontal (with respect to the $U(1)$ connection $\Theta$ ) lifts of circles on $\mathbb{C P}^{5}$ to $S^{5}$ are helices of order 2,3 or 5 . Moreover the order 2 occures iff the first integral (6.45) vanishes. These are the conformal geodesics of $S^{5}$ for which velocity and acceleration are both orthogonal to $K$. A necessary condition is $J(u, a)=0$ (to see it, differentiate $g_{5}(K, a)$ along a conformal geodesic on $S^{5}$ ) which we know can be satisfied, so the conformal geodesics of $\mathbb{C P}^{2}$ with $J(u, a)=0$ come from conformal geodesics of $S^{5}$.

(ii) There is another special case, where the conformal geodesic equations can be explicitly integrated with relative ease. Let $J$ be the complex structure of the ASD Kähler form (6.44), and let $\lambda$ be a non-zero constant. We shall make the ansatz $a=\lambda J(u)$ so that

$$
a_{1}=-\lambda \beta, \quad a_{2}=\lambda \alpha, \quad a_{3}=\lambda \delta, \quad a_{4}=-\lambda \gamma .
$$

Note that the first integral (6.45) is equal to $\lambda$, and

$$
\nabla_{u} a=\lambda J^{2}(u)=-\lambda u, \quad \text { where } \quad \lambda^{2}=|a|^{2}, \quad \text { so that } \quad \tau=1 .
$$

The circle equations (2.4) hold identically as a consequence of the definition of $a$, and can be integrated explicitly. We will not reproduce this calculation here, as we know from Theorem $6 \cdot 2$, that the resulting conformal geodesics lie in the $S U(3)$ orbits of integral curves of $E_{4}$. 


\section{Appendix A}

In this Appendix we establish a non-existence result which narrows down the classification of integrable backgrounds for conformal geodesic equations.

PROPOSITION A·1. There are no non-flat Riemannian Gibbons-Hawking metrics with three commuting Killing vectors.

Proof. Suppose there are, and we have the Gibbons-Hawking metric (3.12) with two further Killing vectors, say $L_{1}, L_{2}$, which commute with each other and with $K=\partial_{\tau}$. We find restrictions on the $L_{1}, L_{2}$ that force flatness. First note that for either $L$

$$
L^{a} \partial_{a}\left(V^{-1}\right)=L^{a} \partial_{a}\left(K^{b} K_{b}\right)=2 L^{a} K^{b} \nabla_{a} K_{b}=2 K^{a} K^{b} \nabla_{a} L_{b},
$$

using $[K, L]=0$, and this is manifestly zero, so that $V$ is constant along each $L$. Decompose each $L$ as

$$
L^{a} \partial_{a}=L^{0} \partial_{\tau}+L^{i} \partial_{i},
$$

then the vanishing of the commutator with $K$ gives

$$
\partial_{\tau} L^{0}=0=\partial_{\tau} L^{i} .
$$

Next with

$$
h_{a b}:=g_{a b}-V K_{a} K_{b}=V \delta_{i j},
$$

where $\delta_{i j}=0$ unless $i, j=1,2,3$ when it is the usual Kronecker delta, we have

$$
\mathcal{L}_{L} h_{a b}=0,
$$

so that $L^{i} \partial_{i}$ is a Killing vector of the flat (Riemannian) 3-metric $\delta_{i j}$. Next, the $(i, j)$ component of

$$
0=\mathcal{L}_{L} g_{a b}=L^{a} \partial_{a} g_{b c}+g_{a c} \partial_{b} L^{a}+g_{b a} \partial_{c} L^{a}
$$

forces $L^{0}$ to be constant when it can be set to zero by subtracting a constant multiple of $K$ from $L$. Finally from the $b=j$ component of

$$
0=\mathcal{L}_{L} K_{b}
$$

we see that

$$
0=L^{i} \partial_{i}\left(V^{-1} \omega_{j}\right)+V^{-1} \omega_{j} \partial_{i} L^{j},
$$

so that $L^{i}$ Lie-drags $\omega$.

Thus any further Killing vector is w.l.o.g. a symmetry of Riemannian flat 3-space, Liedragging both $V$ and $\omega$. These can be translations or rotations. Two independent rotations will not commute so $L_{1}, L_{2}$ can be either two independent translations, in which case we may suppose them orthogonal, or a translation and a rotation, in which case commutation requires them to be orthogonal. There are two cases to consider: 
(i) Suppose $L_{1}=\partial_{x}, L_{2}=\partial_{y}$ then up to constants $V=z$ or $V=1$. Now if $V \neq 1$

$$
d \omega=* d V=d x \wedge d y,
$$

and there is no $\omega$ preserved by both $L_{1}$ and $L_{2}$. Thus $V=1$ when $\omega=0$ and this class of solutions is flat;

(ii) Suppose $L_{1}=\partial_{z}$ and $L_{2}=\partial_{\phi}$ in cylindrical polars. Then $V$ depends only on $\rho$ and is harmonic so up to constants $V=\log \rho$ or $V=1$. Now if $V \neq 1$

$$
d \omega=\frac{* d \rho}{\rho}=d z \wedge d \phi,
$$

and again there is no acceptable solution ${ }^{4}$, except constant $V$.

\section{Appendix B}

In this appendix we shall give necessary and sufficient conditions for a trajectory of a Killing vector in a four-dimensional Einstein space to be a conformal geodesic

PROPOSITION B-1. Let $K$ be a non-null Killing vector on a (pseudo) Riemannian Einstein four manifold $(M, g)$. The trajectories of $K$ are conformal geodesics if and only if

$$
d|K|^{2} \wedge W=0, \text { where } W=\star_{g}(K \wedge d K) .
$$

Proof. Suppose the Killing vector is $K$ with norm $V^{2}=g(K, K)$. We claim that, for any Killing vector

$$
\nabla_{a} K_{b}=\frac{2}{V} V_{[a} K_{b]}+\frac{1}{2 V^{2}} \epsilon_{a b}^{c d} W_{c} K_{d},
$$

for some $W_{a}$ orthogonal to $K$; as a form $W=*(K \wedge d K)$. Now the velocity will be $u^{a}=$ $V^{-1} K^{a}$ and the acceleration as above will be $a_{b}=-V^{-1} \nabla_{b} V$ so we need

$$
\begin{aligned}
-|a|^{2} u_{b}= & u^{c} \nabla_{c} a_{b}=V^{-1} K^{c} \nabla_{c}\left(-V^{-1} V_{b}\right)=-V^{-2} K^{c} \nabla_{b} V_{c}=V^{-2} V^{c} \nabla_{b} K_{c} \\
& =V^{-2} V^{c}\left(\frac{2}{V} V_{[b} K_{c]}+\frac{1}{2 V^{2}} \epsilon_{b c}{ }^{d e} W_{d} K_{e}\right),
\end{aligned}
$$

i.e.

$$
-V^{-3}|\nabla V|^{2} K_{b}=-V^{-3}|\nabla V|^{2} K_{b}+\frac{1}{2} V^{-4} \epsilon_{b}^{c d e} V_{c} W_{d} K_{e},
$$

so the last term needs to vanish, which is equivalent to (B.1).

${ }^{4}$ Note that the claim is not true in the neutral signature: Consider the simplest ASD pp-wave

$$
g=d w d x+d z d y+y^{2} d w^{2},
$$

and complete the square to put it in the Gibbons-Hawking form

$$
g=V\left(d Y d z-\frac{1}{4} d x^{2}\right)+V^{-1}\left(d w+\frac{1}{2}(3 Y)^{-2 / 3} d x\right)^{2},
$$

where $Y=y^{3} / 3$ and $V=(3 Y)^{-2 / 3}$. This metric has a 9D isometry group [5] which is nilpotent, and includes the Abelian 3D algebra spanned by $\left(\partial_{x}, \partial_{w}, \partial_{z}\right)$. 
As a special case we recover the result of [25], where it was shown that if $W=0$, then the Killing trajectories are conformal geodesics. We also find that in the case of the Fubini-Study metric $(6 \cdot 38)$ on $\mathbb{C P}^{2}$ the trajectories of $\partial / \partial \psi$ are conformal geodesics, but the trajectories of $K=\partial / \partial \phi$ (or any other Killing vector) are not.

\section{Appendix C}

In this appendix we shall reformulate the Lorentz force equations, as well as some of the calculations underlying the conformal Killing-Yano tensors in terms of the two component spinor notation [23].

The Hodge $*$ operator of an oriented Riemannian manifold $(M, g)$ is an involution on two-forms, and induces a decomposition

$$
\Lambda^{2}\left(T^{*} M\right)=\Lambda_{+}^{2}\left(T^{*} M\right) \oplus \Lambda_{-}^{2}\left(T^{*} M\right)
$$

of two-forms into self-dual (SD) and anti-self-dual (ASD) components. Locally there exist complex rank-two vector bundles $\mathbb{S}, \mathbb{S}^{\prime}$ over $M$ equipped with covariantly constant symplectic structures $\epsilon, \epsilon^{\prime}$ such that

$$
\mathbb{C} \otimes T M \cong \mathbb{S} \otimes \mathbb{S}^{\prime}, \quad g=\epsilon \otimes \epsilon^{\prime},
$$

and

$$
\Lambda_{+}^{2} \cong \mathbb{S}^{* *} \odot \mathbb{S}^{*}, \quad \Lambda_{-}^{2} \cong \mathbb{S}^{*} \odot \mathbb{S}^{*}
$$

Elements of $\mathbb{S}$ are of the form $\kappa^{A}=\left(\kappa^{0}, \kappa^{1}\right)$, and the spinor indices $A, B, C, \cdots=0,1$ are lowered using the symplectic form $\epsilon_{A B}$ with $\epsilon_{01}=1$. Therefore any two-form admits a spinor decomposition

$$
F_{a b}=f_{A B} \epsilon_{A^{\prime} B^{\prime}}+\tilde{f}_{A^{\prime} B^{\prime}} \epsilon_{A B},
$$

where $f_{A B}=f_{(A B)}$, and $\tilde{f}_{A^{\prime} B^{\prime}}=\tilde{f}_{\left(A^{\prime} B^{\prime}\right)}$. In particular the parallel basis $\left(\Omega^{1}, \Omega^{2}, \Omega^{3}\right)$ of $\Lambda_{+}^{2}$ on a hyper-Kähler four manifold decomposes as

$$
\Omega_{a b}^{i}=\phi_{A^{\prime} B^{\prime}}^{i} \epsilon_{A B}, \quad i=1,2,3 .
$$

Consider the Lorentz force equation (3.7) with self-dual Maxwell field $F$, and set

$$
F_{a b}=|a| \phi_{A^{\prime} B^{\prime}} \epsilon_{A B},
$$

where $\phi_{A^{\prime} B^{\prime}}=\phi_{\left(A^{\prime} B^{\prime}\right)}$ is a valence two symmetric spinor. The Lorentz force equation takes the form

$$
u^{C C^{\prime}} \nabla_{C C^{\prime}} u_{A A^{\prime}}=-|a| \phi_{A^{\prime} B^{\prime}} u_{A}^{B^{\prime}}
$$

and $\phi_{A^{\prime} B^{\prime}} \phi^{A^{\prime} B^{\prime}}=2$. The ASD two-form $W$ from (4.14) is

$$
W_{a b}=\omega_{A B} \epsilon_{A^{\prime} B^{\prime}},
$$

and the CKY equation $(2 \cdot 5)$ takes the form

$$
\nabla_{A A^{\prime}} \omega_{B C}=\epsilon_{A(B} K_{C) A^{\prime}}, \quad \text { where } \quad K=\partial / \partial \psi .
$$


First note that $\omega_{A B}$ gives rise to three first integrals of the geodesic equations. To see this consider the quadratic expression in velocity $\omega_{A B} \phi_{A^{\prime} B^{\prime}}^{i} u^{A A^{\prime}} u^{B B^{\prime}}$, whose rate of change is

$$
u^{C C^{\prime}} \nabla_{C C^{\prime}}\left(\omega_{A B} \phi_{A^{\prime} B^{\prime}}^{i} u^{A A^{\prime}} u^{B B^{\prime}}\right)=\frac{1}{2} u^{A A^{\prime}} \phi_{A^{\prime} B^{\prime}}^{i} K_{A}^{B^{\prime}}=\frac{1}{2} u^{A A^{\prime}} \nabla_{A A^{\prime}} x^{i},
$$

by (C1) and (3.13) so that three constants of the motion for the geodesic equation are defined by

$$
q^{i}:=\omega_{A B} \phi_{A^{\prime} B^{\prime}}^{i} u^{A A^{\prime}} u^{B B^{\prime}}-\frac{1}{2} x^{i} .
$$

It is known from [25] that a Killing spinor will give constants of the motion for the conformal geodesic equation: consider $\omega_{A^{\prime} B^{\prime}} u^{A A^{\prime}} a_{A}^{B^{\prime}}$ then its rate of change following (1.1) and the definition $a=\nabla_{u} u$ is

$$
u^{C C^{\prime}} \nabla_{C C^{\prime}}\left(\omega_{A B} u^{A A^{\prime}} a_{A^{\prime}}^{B}\right)=-\frac{1}{2} K_{b} a^{b}=-\frac{1}{2} u^{c} \nabla_{c}\left(K_{b} u^{b}\right),
$$

so that the following is a conserved quantity for the conformal geodesic equation:

$$
\omega_{A B} u^{A A^{\prime}} a_{A^{\prime}}^{B}+\frac{1}{2} K_{b} u^{b} .
$$

In the present case, when we have the relation

$$
u_{A A^{\prime}} a_{B^{\prime}}^{A}=u_{A\left(A^{\prime}\right.} a_{\left.B^{\prime}\right)}^{A}=-\frac{1}{2}|a| \phi_{A^{\prime} B^{\prime}}
$$

for a real constant spinor $\phi_{A^{\prime} B^{\prime}}$ between velocity and acceleration, this constant becomes

$$
-|a| u^{A A^{\prime}} u^{B B^{\prime}} \phi_{A^{\prime} B^{\prime}} \omega_{A B}+\frac{1}{2} K_{b} u^{b} .
$$

This is the first integral $\mathcal{W}$ in $(4 \cdot 17)$.

Acknowledgements. The work of MD has been partially supported by STFC consolidated grants ST/P000681/1, and ST/T000694/1.

\section{REFERENCES}

[1] T. ADACHI. (1995) Kähler magnetic flows for a manifold of constant holomorphic sectional curvature. Tokyo J. Math. 18, 473-483.

[2] T. AdAChi, S. Maeda and S. Udagawa. Circles in a complex projective space. Osaka J. Math. 32 (1995), 709-719.

[3] T. N. BAiley and M. G. EAstwood. Conformal circles and parametrizations of curves in conformal manifolds. Proc. Amer. Math. Soc. 108 (1990), 215-221.

[4] T. N. Bailey, M. G. EASTwood and A. R. Gover. Thomas's structure bundle for conformal, projective and related structures. Rocky Mountain J. Math. 24 (1994), 1191-1217.

[5] S. CASEY, M. DUNAJSKI and K. P. TOD. Twistor geometry of a pair of second order ODEs Comm. Math. Phys. 321 (2013), 681-701.

[6] A. Comtet. On the Landau levels on the hyperbolic plane. Ann. of Phys. 173 (1987), 185-209.

[7] M. DunAJSKI. Solitons Instantons and Twistors. Oxford Graduate Texts in Math. (Oxford University Press, 2009).

[8] M. DUNAJSKI and W. KRYŃSKI. Variational principles for conformal geodesics. (2021) arXiv: arXiv:2104.13105.

[9] M. DunAJSKI and K. P. Tod. Four dimensional metrics conformal to Kahler. Math. Proc. Camb. Phil. Soc. 148 (2010), 485.

[10] M. DUNAJSKI and P. TOD. Self-dual conformal gravity. Comm. Math. Phys. 331 (2014), 351-373. 
[11] T. Eguchi and A. Hanson. Selfdual solutions to Euclidean gravity. Annals of Phys 120 (1979), $82-105$.

[12] V. P. Frolov, P. Krtous, and D. KubiznaK. Black holes, hidden symmetries and complete integrability. Living Revievs in Relativity (2017).

[13] G. W. GibBons and S. W. HaW KING. Gravitational multi - instantons. Phys. Lett. B78 (1978), 430.

[14] G. W. GibBons and C. Pope. $\mathbb{C P}^{2}$ As a gravitational instanton. Commun. Math. Phys. 61 (1978), 239.

[15] G. W. Gibbons and P. J. Ruback. The hidden symmetries of multicentre metrics. Comm. Math. Phys. 115 (1988), 267-300.

[16] A. R. Gover, D. Snell and A. Taghavi-Chabert. Distinguished curves and integrability in Riemannian, conformal and projective geometry (2018) arXiv:1806.09830.

[17] T. Houri, O. TAKeshi and Y. YUKINORI. Closed conformal Killing-Yano tensor and geodesic integrability. J. Phys. A41. (2007), 025204.

[18] F. JOACHIMSTHAL. Observationes de lineis brevissimis et curvis curvaturae in superficiebus secundi gradus JRAM 26 (1843), 155-171.

[19] L. D. LANDAU and E. Lifshitz. The Classical Theory of Fields (Pergamon Press, 1962).

[20] S. MAEDA and T. ADACHI. Integral curves of Killing vector fields in a complex projective space. Mem. Fac. Sci. Eng. Shimane Univ. B34 (2001), 61-85.

[21] S. Maeda and Y. OHnita. Helical geodesic immersion into complex space forms. Geom. Dedicata 30 (1989), 93-114.

[22] K. NomizU and K. YANo. On circles and spheres in Riemannian geometry. Math. Ann. 210 (1974), $163-170$.

[23] R. PENRose and W. RINDler. Spinors and Space-time. Two-Spinor Calculus and Relativistic Fields. Cambridge Monogr. Math. Phys. (Cambridge University Press, Cambridge, 1987, 1988).

[24] J. SiHLAN and V. ZADNIK Conformal theory of curves with tractors. (2018), arXiv:1805.00422.

[25] K. P. ToD. Some examples of the behaviour of conformal geodesics. J. Geom. Phys. 62 (2012), $1778-1792$.

[26] G. Valent. Integrability Versus Separability for the Multi-Centre Metrics. Comm. Math. Phys. 244 (2004), 571-594. 